\title{
Unsteady turbulent plume models
}

Scase, M.M. and Hewitt, R.E.

2012

MIMS EPrint: 2013.59

Manchester Institute for Mathematical Sciences

School of Mathematics

The University of Manchester

\footnotetext{
Reports available from: http://eprints.maths.manchester.ac.uk/

And by contacting: The MIMS Secretary

School of Mathematics

The University of Manchester

Manchester, M13 9PL, UK
} 


\title{
Unsteady turbulent plume models
}

\author{
M. M. Scase ${ }^{1} \dagger$ and R. E. Hewitt ${ }^{2}$ \\ ${ }^{1}$ Faculty of Engineering, University of Nottingham, Nottingham NG7 2RD, UK \\ 2 School of Mathematics, The University of Manchester, Oxford Road, Manchester M13 9PL, UK \\ (Received 19 August 2011; revised 28 November 2011; accepted 3 February 2012; \\ first published online 12 March 2012)
}

Four existing integral models of unsteady turbulent plumes are revisited. We demonstrate that none of these published models is ideal for general descriptions of unsteady behaviour and put forward a modified model. We show that the most recent (top-hat) plume model (Scase et al. J. Fluid Mech., vol. 563, 2006, p. 443), and the earlier (Gaussian) plume models (Delichatsios J. Fluid Mech., vol. 93, 1979, p. 241; Yu Trans. ASME, vol. 112, 1990, p.186), are all ill-posed. This ill-posedness arises from the downstream growth of short-scale waves, which have an unbounded downstream growth rate. We show that both the top-hat and the Gaussian (Yu) models can be regularized, rendering them well-posed, by the inclusion of a velocity diffusion term. The effect of including this diffusive mechanism is to include a vertical structure in the model that can be interpreted as representing the vertical extent of an eddy. The effects of this additional mechanism are small for steady applications, and cases where the plume forcing can be considered to follow a power law (both of which have been studied extensively). However, the inclusion of diffusion is shown to be crucial to the general initial-value problem for unsteady models.

Key words: plumes/thermals

\section{Introduction}

Models of steady turbulent plumes are well-known; more recently however the analogous flows driven by unsteady source conditions have received some attention. There are many applications that would benefit from an improved understanding of such flows including, but not limited to, volcanic eruption modelling, industrial accident planning, oil fire modelling and sea-ice evolution modelling; see for example Holland (2011) and the recent review articles by Kaye (2008) and Hunt \& van den Bremer (2010).

The recent model due to Scase et al. (2006b) (herein referred to as SCDH) developed the ideas of the classical Morton, Taylor \& Turner (1956) model to allow for fully unsteady source conditions at the base of a top-hat plume. Subsequent work has sought to verify the model via laboratory experiments (e.g. Scase, Caulfield \& Dalziel 2008) and large-scale numerical simulations (e.g. Scase, Aspden \& Caulfield 2009). Previous authors, in modelling fire plumes, have developed similar models that predate that of SCDH, but under the assumption of a Gaussian distribution of the plume properties over the radius of the plume. These models have also received some

$\dagger$ Email address for correspondence: matthew.scase@nottingham.ac.uk 
experimental validation. The application of these earlier models has been primarily restricted to cases where the source buoyancy flux follows a power law in time, for which similarity solutions can be found.

Given the wide range of applications for unsteady turbulent plume models, and the number of integral models available, the present authors have returned to the origins of these different models to consider which are most appropriate. In particular the top-hat model of SCDH, and the Gaussian models of Delichatsios (1979), Yu (1990), and Vul'fson \& Borodin (2001) are discussed herein.

A 'steady plume' is only steady in the sense that its ensemble average is steady. Any real plume, be it existing in nature or the laboratory, will be subject to small perturbations in the source conditions due to the plume's turbulent nature. We therefore begin our assessment of the unsteady models by linearizing about steady solutions and describing the response to (asymptotically) small fluctuations in the source conditions.

By considering the evolution of such small harmonic fluctuations we will show that the three unsteady models of Delichatsios (1979) and Yu (1990), and SCDH are all illposed. The model of Vul'fson \& Borodin (2001) only remains well-posed by enforcing an extremely strong assumption, namely that the plume remains conical at all times, which must render the value of this model for general applications questionable. The ill-posedness of these models arises through the development of high-frequency/shortwavelength fluctuations that grow asymptotically rapidly with downstream distance. These short-wave modes arise as a consequence of the artificial modelling of each (infinitesimally thin) plume cross-section as being divorced from the neighbouring cross-sections, thereby allowing asymptotically small vertical length scales to develop. To counter this, we propose that the models require an additional physical effect, which communicates information vertically, and we put forward a velocity diffusion as the most likely mechanism to achieve these aims.

We suggest that the vertical structure of eddies comprising the plume is significant in preventing the formation of arbitrarily small length scales. Motivated by the structure of the eddies in the plume we include a diffusion term in the momentum flux equation, and the precise form of the term is found by appealing to Prandtl's mixing length theory. We demonstrate that the addition of this term to the classical model (Morton et al. 1956) has only a small effect on the steady solutions, but is nonetheless sufficient to remove the ill-posedness in a subset of the existing unsteady models.

The ill-posed nature of these model equations has remained undiscovered for some time, in large part due to previous authors restricting attention to numerical solutions of ordinary differential equations that arise from similarity reductions of the full model equations. Where the initial-value problem has been addressed, it has been through first-order schemes with low resolution. Unfortunately the inherent numerical diffusion in these numerical approaches concealed the ill-posedness of the model, and led to results being presented that were necessarily unconverged.

By considering a single-mode harmonic perturbation to the models discussed above, we will demonstrate their ill-posedness analytically and subsequently verify these results using a numerical scheme for the equivalent linearized system. We then proceed to introduce an improved and well-posed model and describe a second-orderaccurate numerical scheme for tackling the resulting nonlinear initial-value problem. 


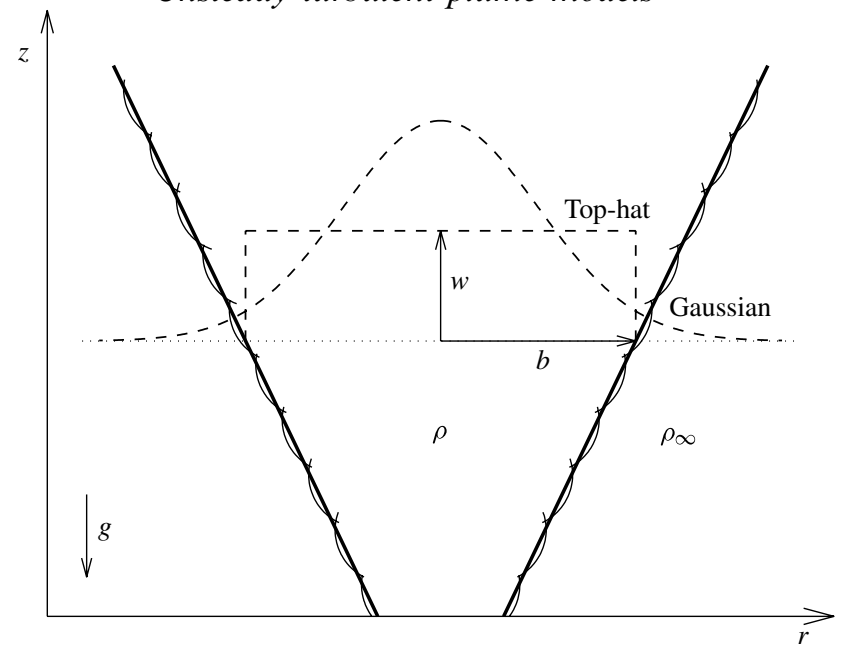

FIGURE 1. Schematic illustration of a typical plume.

\subsection{Notation}

Figure 1 is a schematic diagram of a plume and introduces the notation used throughout the present treatment. The top-hat plume radius and vertical velocity are denoted respectively by $b(z, t)$ and $w(z, t)$. The density of the plume fluid is denoted $\rho(z, t)$. 'Top-hat' indicates that there is no radial dependence on these quantities and the variables may be regarded as radially averaged quantities from an ensemble average of plume realizations. Gravity acts in the direction of decreasing $z$ and the density of the ambient fluid is taken to be a constant, $\rho_{\infty}$. We define a mass flux, $\pi Q$, momentum flux, $\pi M$, and buoyancy flux, $\pi F$, in the following manner:

$$
Q=\rho b^{2} w, \quad M=\rho b^{2} w^{2}, \quad F=g\left(\rho_{\infty}-\rho\right) b^{2} w .
$$

In the Boussinesq limit as $\rho \rightarrow \rho_{\infty}$ and $g \rightarrow \infty$, keeping $g^{\prime}=g\left(\rho_{\infty}-\rho\right) / \rho$ fixed, we may write

$$
Q=\rho_{\infty} b^{2} w, \quad M=\rho_{\infty} b^{2} w^{2}, \quad F=\rho_{\infty} g^{\prime} b^{2} w,
$$

and

$$
b=\frac{Q}{\sqrt{\rho_{\infty} M}}, \quad w=\frac{M}{Q}, \quad g^{\prime}=\frac{F}{Q} .
$$

For convenience we define the vector $\boldsymbol{Q}=(Q, M, F)^{\top}$ and similarly for $\boldsymbol{Q}_{B}$ and $\boldsymbol{q}$ (to be defined later).

\subsection{Layout of the paper}

The format of this paper is as follows. In $\S 2$ we return to the unsteady 'top-hat plume model' of SCDH and show that this model system is susceptible to highfrequency/short-wave linear instabilities and as such is an ill-posed problem. In this same section we propose the inclusion of a diffusion term that eliminates the highfrequency instability, leading to a well-posed and reproducible numerical initial-value problem. Section 3 presents numerical results for this new model, showing that it is stable to linear harmonic disturbances, and describes the nonlinear evolution of the top-hat plumes. In $\S 4$ we review Gaussian plume models of Delichatsios (1979) 
and Yu (1990) and show that the ill-posedness of the simpler top-hat model can be found in these cases too. In $\S 5$ we revisit similarity solutions of unsteady plume models investigated by previous authors and their empirical validations. We see that the differences between all the models, both with and without diffusion, are small, and of the order of the experimental error.

\section{Unsteady top-hat plume models}

We begin our discussion by considering the temporal development of the 'top-hat' plumes described by SCDH, their equation 2.16 . The plume is governed by the system

$$
\begin{gathered}
\frac{\partial}{\partial t}\left(\frac{Q^{2}}{M}\right)+\frac{\partial Q}{\partial z}=2 \alpha \rho_{\infty}^{1 / 2} M^{1 / 2}, \\
\frac{\partial Q}{\partial t}+\frac{\partial M}{\partial z}=\frac{Q F}{M}, \\
\frac{\partial}{\partial t}\left(\frac{Q F}{M}\right)+\frac{\partial F}{\partial z}=0 .
\end{gathered}
$$

In this system $\alpha$ is the plume entrainment coefficient, which is assumed constant. For a detailed derivation of these equations we refer the reader to SCDH.

We will demonstrate below that there is an inherent difficulty associated with the model equations (2.1); the initial-value problem is ill-posed. This ill-posedness manifests itself as a short-wavelength/high-frequency instability with an unbounded growth rate, in the manner of the problems reviewed by Jospeh \& Saut (1990). Although we begin with a discussion of the top-hat model, such features are not exclusive to this choice of plume model.

\subsection{Difficulties associated with unsteady top-hat models}

A general approach is to consider the response of the unsteady plume model (2.1) to infinitesimal perturbations of the source conditions and then address their temporal and spatial development 'downstream'. A (well-known) steady basic state $\boldsymbol{Q}_{B}(z)$, due to Morton et al. (1956), exists with

$$
\begin{gathered}
Q_{B}(z)=\frac{3}{5}\left(\frac{9}{20}\right)^{1 / 3}\left(2 \alpha \rho_{\infty}^{1 / 2}\right)^{4 / 3} F_{0}^{1 / 3} z^{5 / 3}, \\
M_{B}(z)=\left(\frac{9}{20}\right)^{2 / 3}\left(2 \alpha \rho_{\infty}^{1 / 2}\right)^{2 / 3} F_{0}^{2 / 3} z^{4 / 3}, \\
F_{B}(z)=F_{0} .
\end{gathered}
$$

We may write $\boldsymbol{Q}_{B}=\left(c_{Q} z^{5 / 3}, c_{M} z^{4 / 3}, F_{0}\right)^{\top}$ defining the dimensional constants $c_{Q}$ and $c_{M}$ (as above) and further we write the steady velocity field as $w_{B}=M_{B} / Q_{B}=c_{w} z^{-1 / 3}$ for a dimensional constant $c_{w}=c_{M} / c_{Q}$. We perturb this solution via

$$
\left.\begin{array}{l}
Q=Q_{B}(z)[1+\delta q(z, t)], \quad M=M_{B}(z)[1+\delta m(z, t)], \\
F=F_{B}(z)[1+\delta f(z, t)],
\end{array}\right\}
$$

where $\delta \ll 1$ is a disturbance amplitude and we can define $\boldsymbol{q}=(q, m, f)^{\top}$.

Rather than examining the linear initial-value problem for $\boldsymbol{q}(z, t)$ we Fourier decompose and consider a single harmonic of frequency $\omega$, such that $\boldsymbol{q}(z, t)=$ $\hat{\boldsymbol{q}}(z) \exp (\mathrm{i} \omega t)$. It proves convenient to make the change of variables $\zeta=\left(z \omega / w_{B}\right)^{1 / 2}$, 
$\tau=\omega t$, where $\zeta$ and $\tau$ are dimensionless. Given this transformation, the downstream evolution of the perturbation is governed by

$$
\mathrm{i} \omega\left(\begin{array}{rrr}
2 & -1 & 0 \\
1 & 0 & 0 \\
1 & -1 & 1
\end{array}\right) \hat{\boldsymbol{q}}+\frac{2}{3 \zeta} \frac{\mathrm{d} \hat{\boldsymbol{q}}}{\mathrm{d} \zeta}+\frac{1}{3 \zeta^{2}}\left(\begin{array}{rrr}
5 & -\frac{5}{2} & 0 \\
-4 & 8 & -4 \\
0 & 0 & 0
\end{array}\right) \hat{\boldsymbol{q}}=\mathbf{0} .
$$

For definiteness we shall assume that the perturbation is forced solely via the buoyancy flux at the source, that is $f(0, \tau)=\exp \{i \tau\}$ (i.e. $\hat{f}(0)=1$ ), whilst the mass flux and momentum flux perturbations at the source remain zero. The downstream evolution can be combined into a single higher-order equation for the buoyancy flux:

$$
\frac{\mathrm{d}^{3} \hat{f}}{\mathrm{~d} \zeta^{3}}+\frac{11+9 \mathrm{i} \zeta^{2}}{2 \zeta} \frac{\mathrm{d}^{2} \hat{f}}{\mathrm{~d} \zeta^{2}}-\frac{2\left(27 \zeta^{4}-8\right)-153 \mathrm{i} \zeta^{2}}{8 \zeta^{2}} \frac{\mathrm{d} \hat{f}}{\mathrm{~d} \zeta}-3 \frac{87 \zeta^{2}+2 \mathrm{i}\left(9 \zeta^{2}-20\right)}{\zeta} \hat{f}=0
$$

Rotating and stretching coordinates in the Argand plane via $\Lambda=$ $(15 / 8)^{1 / 2} \exp \{-\mathrm{i} \pi / 4\} \zeta$, and defining $\hat{f}_{\star}=\hat{f} \exp \left\{-2 \Lambda^{2} / 5\right\}$, we can rewrite $(2.5)$ as

$$
\frac{\mathrm{d}^{3} \hat{f}_{\star}}{\mathrm{d} \Lambda^{3}}+\frac{11}{2 \Lambda} \frac{\mathrm{d}^{2} \hat{f}_{\star}}{\mathrm{d} \Lambda^{2}}+\frac{\Lambda^{2}+2}{\Lambda^{2}} \frac{\mathrm{d} \hat{f}_{\star}}{\mathrm{d} \Lambda}+\frac{2 \hat{f}_{\star}}{\Lambda}=0 .
$$

Equation (2.6) admits solutions in terms of the special Struve-H function, $\mathscr{H}$, and the Bessel function of the first kind, $\mathscr{J}$ (Abramowitz \& Stegun 1965). Applying the condition $\hat{f}(0)=1$, we obtain

$$
\begin{aligned}
\hat{f}= & \frac{3}{8} \mathrm{e}^{2 \Lambda^{2} / 5}\left\{\left(\frac{2 \pi^{2}}{\Lambda^{13}}\right)^{1 / 4} \Gamma\left(\frac{3}{4}\right)\left[\left(3+4 \Lambda^{2}\right) \mathscr{H}_{1 / 4}(\Lambda)-6 \Lambda \mathscr{H}_{5 / 4}(\Lambda)\right]\right. \\
& \left.+\frac{c}{\Lambda^{5 / 4}} \mathscr{J}_{7 / 4}(\Lambda)-\frac{4}{\Lambda^{2}}\right\}
\end{aligned}
$$

for arbitrary constant $c$, where $\Gamma$ is the usual gamma function. Substituting back to find $\hat{q}$, and $\hat{m}$ together with the conditions that both remain bounded as $\zeta \rightarrow 0$ yields that $c \equiv 0$ and therefore the solutions to (2.4) are given by

$$
\begin{aligned}
q= & \frac{3}{32} \mathrm{e}^{2 \Lambda^{2} / 5+\mathrm{i} \tau}\left\{\frac { 1 } { \Lambda ^ { 2 } } ( \frac { 2 \pi ^ { 2 } } { \Lambda ^ { 1 3 } } ) ^ { 1 / 4 } \Gamma ( \frac { 3 } { 4 } ) \left[\left(16 \Lambda^{4}+2 \Lambda^{2}-15\right) \mathscr{H}_{1 / 4}(\Lambda)\right.\right. \\
& \left.\left.-2 \Lambda\left(22 \Lambda^{2}-15\right) \mathscr{H}_{5 / 4}(\Lambda)\right]-\frac{4\left(4 \Lambda^{2}-5\right)}{\Lambda^{4}}\right\}, \\
m= & \frac{1}{16} \mathrm{e}^{2 \Lambda^{2} / 5+\mathrm{i} \tau}\left\{\frac { 3 ( 5 + 2 \Lambda ^ { 2 } ) } { \Lambda ^ { 2 } } ( \frac { 2 \pi ^ { 2 } } { \Lambda ^ { 1 3 } } ) ^ { 1 / 4 } \Gamma ( \frac { 3 } { 4 } ) \left[\left(3+4 \Lambda^{2}\right) \mathscr{H}_{1 / 4}(\Lambda)\right.\right. \\
& \left.\left.-6 \Lambda \mathscr{H}_{5 / 4}(\Lambda)\right]-\frac{4\left(15+16 \Lambda^{2}\right)}{\Lambda^{4}}\right\}, \\
f & =\frac{3}{8} \mathrm{e}^{2 \Lambda^{2} / 5+\mathrm{i} \tau}\left\{( \frac { 2 \pi ^ { 2 } } { \Lambda ^ { 1 3 } } ) ^ { 1 / 4 } \Gamma ( \frac { 3 } { 4 } ) \left[\left(3+4 \Lambda^{2}\right) \mathscr{H}_{1 / 4}(\Lambda)\right.\right. \\
& \left.\left.-6 \Lambda \mathscr{H}_{5 / 4}(\Lambda)\right]-\frac{4}{\Lambda^{2}}\right\} .
\end{aligned}
$$




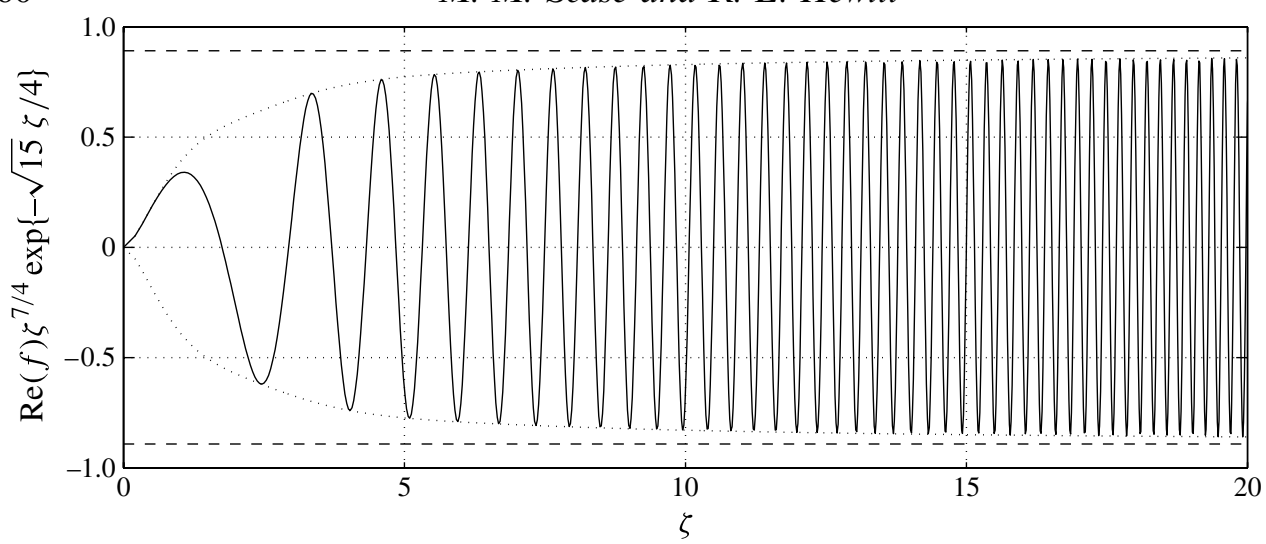

FIGURE 2. The downstream development of a linear harmonic perturbation to the otherwise steady plume flow. The image shows the perturbation $f$ scaled according to the leading-order far-downstream asymptotic form versus $\zeta$. The horizontal dashed lines show the limiting extent $(2 / 5) 2^{3 / 8} 15^{1 / 8} \Gamma(3 / 4)$.

For large values of $\zeta$, and hence $\Lambda$, we may make use of the asymptotic form

$$
\mathscr{H}_{a}(\Lambda) \sim \sqrt{\frac{2}{\pi \Lambda}} \sin \left(\Lambda-\frac{a \pi}{2}-\frac{\pi}{4}\right)+\frac{1}{\sqrt{\pi} \Gamma\left(a+\frac{1}{2}\right)}\left(\frac{\Lambda}{2}\right)^{a-1}+O\left(\Lambda^{-3 / 2}\right),
$$

noting that $a>-1 / 2$ in $(2.8)$, to show that

$$
\begin{aligned}
f \sim & \frac{2 \times 2^{3 / 8} \times 15^{1 / 8}}{5} \Gamma\left(\frac{3}{4}\right) \frac{\mathrm{e}^{\sqrt{15} \zeta / 4}}{\zeta^{7 / 4}} \\
& \times \exp \left\{\frac{\mathrm{i}}{4}\left[4 \tau+\sqrt{15} \zeta-3 \zeta^{2}-\frac{7 \pi}{4}\right]\right\}\left[1+O\left(\frac{1}{\zeta}\right)\right] .
\end{aligned}
$$

The development of the downstream disturbance is illustrated in figure 2. The real part of the perturbation is shown, normalized by the growth terms in (2.10), $\zeta^{-7 / 4} \exp \{\sqrt{15} \zeta / 4\}$. The dotted lines indicate the absolute value of $f$, and the dashed lines show the asymptotic limiting extent of the normalized perturbation, $(2 / 5) 2^{3 / 8} 15^{1 / 8} \Gamma(3 / 4)$. We have verified that the analytical solution derived above and the numerical solution of the linearized system (2.4) are in agreement.

It follows that the perturbation is a wave propagating downstream with the plume, as can be seen from the dominant contribution to the exponential term:

$$
\exp \left\{\frac{\mathrm{i}}{4}\left[4 \tau-3 \zeta^{2}\right]\right\}=\exp \left\{\mathrm{i} \omega\left[t-\frac{3}{4} \frac{z}{w_{B}(z)}\right]\right\}=\exp \left\{\mathrm{i} \omega\left[t-\frac{3}{4 c_{w}} z^{4 / 3}\right]\right\} .
$$

The phase speed of the wave represented by (2.11) is therefore also $c_{w} z^{-1 / 3}$, which is the vertical velocity of the plume.

The amplitude of the wave grows as $\zeta^{-7 / 4} \exp \{\sqrt{15} \zeta / 4\}$ for large $\zeta$. It can be seen therefore that the solutions grow in magnitude and ultimately the asymptotic assumption that $q, m, f \sim O(1)$ fails. We observe that, in the far field, the exponential downstream growth of the wave is $\exp \left\{O\left(\omega^{1 / 2} z^{2 / 3}\right)\right\}$, and the wavelength of the 
perturbation is

$$
\lambda=\frac{2 \pi}{\omega} c_{w} z^{-1 / 3} .
$$

A similar short-scale high-frequency wave has recently been described for pulsatile jets by Hewitt \& Duck (2011).

This growth of downstream perturbations is not simply indicating a linear instability of the steady base flow, it demonstrates that the unsteady evolution of (2.1) is fundamentally an ill-posed problem. This is because there is no finite dominant frequency and any arbitrarily large downstream growth rate can be achieved by choosing a suitably small-scale/high-frequency perturbation. The coefficient of exponential downstream growth is proportional to $\omega^{1 / 2}$ and corresponds to waves with a wavelength proportional to $\omega^{-1}$. Increasing the spatio-temporal resolution of any numerical scheme resolves more of the unstable spectrum present in the initial conditions and leads to different results; converged numerical results are unachievable. The influence of these modes is particularly strongly felt for discontinuous changes in the source conditions, owing to the slower (algebraic rather than exponential) decay of the Fourier amplitudes at high frequencies.

\subsection{Regularization of the unsteady top-hat model}

One might conclude that the model equations are without value based upon their illposed nature. However as noted in Jospeh \& Saut (1990), one may equally conclude that the model is missing a physical process that dominates at short wavelengths. Given the relative success of the steady model and the recent investigations of the unsteady extension (e.g. Scase et al. 2008, 2009) we propose that the unsteady model does have a predictive value but requires regularizing to render it well-posed. The ill-posed nature of the system (2.1) was not realized in SCDH due to the method of numerical solution (see Appendix therein). The first-order scheme's inherent numerical diffusion removed the short-wavelength instabilities for the spatio-temporal resolutions chosen.

One likely mechanism for regularization of the model is to introduce a downstream diffusion of velocity. It is straightforward to include an additional diffusion term in the momentum equation for the plume, with a local diffusion coefficient of $\hat{\kappa}$. Appealing to Prandtl's mixing-length theory we pose that the diffusion coefficient should be proportional to a local length scale and local velocity, hence we take $\hat{\kappa}=\varepsilon b w / 2 \alpha$ where $\varepsilon$ is dimensionless. Now repeating the derivation of SCDH but including an extra diffusion term, we find that the momentum equation $((2.1 c)$ in their work) becomes

$$
\frac{\mathrm{D} w}{\mathrm{D} t}=g \frac{\rho_{\infty}-\rho}{\rho}+\frac{2 u_{e} w}{b}+\frac{\varepsilon}{2 \alpha} \frac{\partial}{\partial z}\left(b w \frac{\partial w}{\partial z}\right) .
$$

(This differs from the approach taken by Schmidt 1941 who included radial gradients of velocity in the momentum equation when exploiting Prandtl's mixing-length theory to characterize entrainment.) Following the approach of SCDH further, we can integrate over the plume width to once again obtain $(2.1 b)$ herein, but now including diffusion of the velocity field:

$$
\frac{\partial Q}{\partial t}+\frac{\partial M}{\partial z}=\frac{Q F}{M}+\varepsilon \frac{Q^{2}}{2 \alpha \rho_{\infty}^{1 / 2} M} \frac{\partial}{\partial z}\left[M^{1 / 2} \frac{\partial}{\partial z}\left(\frac{M}{Q}\right)\right] .
$$


The purpose of including the diffusion term in (2.14) is to regularize the unsteady model by removing the increasing downstream spatial growth of linear disturbance modes of high frequency and short wavelength. Note that any positive value of $\varepsilon$ is sufficient to regularize the unsteady model as for any $\varepsilon>0$ there will exist a height at which the perturbation's wavelength will have decreased to such an extent that diffusion will dominate the dynamics. However, given how established the classical model is, any plausible modification of the governing system should not greatly affect the steady solution structure. The steady form of (2.1) with (2.1b) replaced by (2.14) still supports steady point-source solutions of the form $\boldsymbol{Q}=\hat{\boldsymbol{Q}}_{B}$ where

$$
\begin{gathered}
\hat{Q}_{B}(z)=Q_{B}(z)\left(1-\frac{\varepsilon}{10}\right)^{-1 / 3}=c_{\hat{Q}} z^{5 / 3}, \\
\hat{M}_{B}(z)=M_{B}(z)\left(1-\frac{\varepsilon}{10}\right)^{-2 / 3}=c_{\hat{M}} z^{4 / 3}, \\
\hat{F}_{B}(z)=F_{0},
\end{gathered}
$$

and $\hat{\imath}$ denotes a quantity pertaining to the regularized model. This yields the, perhaps surprising, result that the inclusion of the diffusion term does not affect the expected plume radius profile of $b=6 \alpha z / 5$. Furthermore, provided that $\varepsilon$ is small, the velocity and reduced gravity are only affected to order $\varepsilon$, specifically

$$
\begin{gathered}
\hat{w}_{B}=\frac{5}{3}\left(\frac{9}{20}\right)^{1 / 3}\left(2 \alpha \rho_{\infty}^{1 / 2}\right)^{-2 / 3} F_{0}^{1 / 3} z^{-1 / 3}\left(1-\frac{\varepsilon}{10}\right)^{-1 / 3}=c_{\hat{w}} z^{-1 / 3}, \\
\hat{g}_{B}^{\prime}=\frac{5}{3}\left(\frac{9}{20}\right)^{-1 / 3}\left(2 \alpha \rho_{\infty}^{1 / 2}\right)^{-4 / 3} F_{0}^{2 / 3} z^{-5 / 3}\left(1-\frac{\varepsilon}{10}\right)^{1 / 3} .
\end{gathered}
$$

A common technique for measuring the entrainment coefficient, $\alpha$, is to analyse the spreading angle of an observed plume radius. Hence, we see that the rôle of the diffusion in the steady model is relatively unimportant in this regard. However, the diffusion is crucial in preventing growth of linear high-frequency instabilities in the unsteady model.

An alternative approach to regularizing the unsteady model would be to utilize the method of Kaminski, Tait \& Carazzo (2005, see (3.3)-(3.5) in their treatment) but including the unsteady terms in the Reynolds-averaged equations. That approach has not been pursued here.

\subsubsection{Interpretation as an eddy diffusion}

By appealing to Prandtl's mixing-length theory in introducing the velocity diffusion term above, in (2.13), we are appealing to ideas based on the concept of eddy diffusion. The diffusion term scales locally with the plume width and mean velocity and so varies depending on the local structure of the plume, which is characterized by the eddies. However, given the strong assumptions made in invoking the entrainment assumption it is not clear that the introduction of the diffusive term in (2.13) represents an eddy diffusion of velocity in the rigorous sense. If we were to interpret it as such, a natural follow-on question would be that since the buoyancy is being acted upon by the same eddies that are diffusing the momentum, should not a buoyancy diffusion term be included in the regularizing model too. It is straightforward to include a similar buoyancy diffusion term to the velocity diffusion term and in the 
steady case we have

$$
\begin{gathered}
\frac{\mathrm{d} Q}{\mathrm{~d} z}=2 \alpha \rho_{\infty}^{1 / 2} M^{1 / 2}, \quad \frac{\mathrm{d} M}{\mathrm{~d} z}=\frac{Q F}{M}+\varepsilon \frac{Q^{2}}{2 \alpha \rho_{\infty}^{1 / 2} M} \frac{\mathrm{d}}{\mathrm{d} z}\left[M^{1 / 2} \frac{\mathrm{d}}{\mathrm{d} z}\left(\frac{M}{Q}\right)\right], \\
\frac{\mathrm{d} F}{\mathrm{~d} z}=K \frac{Q^{2}}{2 \alpha \rho_{\infty}^{1 / 2} M} \frac{\mathrm{d}}{\mathrm{d} z}\left[M^{1 / 2} \frac{\mathrm{d}}{\mathrm{d} z}\left(\frac{F}{Q}\right)\right],
\end{gathered}
$$

where we have introduced a buoyancy diffusion coefficient $K$. Power-law solutions of (2.17) can be found and are given by

$$
\begin{gathered}
Q=\frac{3 c}{5}\left(\frac{9}{20}\right)^{1 / 3} 2^{4 / 3} F_{0}^{1 / 3}\left(1-\frac{\varepsilon}{10}\right)^{-1 / 3} z^{(5 / 3)[1+\Phi / 5]}, \\
M=c^{2}\left(\frac{9}{20}\right)^{2 / 3}\left(1+\frac{\Phi}{5}\right)^{2} 2^{2 / 3} F_{0}^{2 / 3}\left(1-\frac{\varepsilon}{10}\right)^{-2 / 3} z^{(4 / 3)[1+\Phi / 2]}, \quad F=F_{0} z^{\Phi} \quad(2.18 b, c)
\end{gathered}
$$

(cf. (2.2)) where we have taken, without loss of generality, $\alpha \rho_{\infty}^{1 / 2}=1$, and $\Phi$ is given by

$$
\Phi=\frac{\left\{25+130 K+K^{2}\right\}^{1 / 2}-(5+9 K)}{2(1-2 K)} \sim 2 K+O\left(K^{2}\right) .
$$

By substitution into $(2.17 b)$, the constant $c$ can be found explicitly as the root of a cubic equation with no linear or quadratic terms and is given asymptotically by $c \sim 1-13 K / 15+O\left(\varepsilon^{2}, \varepsilon K, K^{2}\right)$.

This solution has the undesirable property of not following the established classical power-law solutions, unlike (2.15), and drifts further away from these solutions for increasing $K$.

We anticipate that any diffusion acting will be weak, only affecting small wavelengths to any significant degree. Hence, given the uncertainty surrounding the rigour of identifying (2.17) as a genuine implementation of a consistent eddy diffusion model and the added difficulty of introducing another parameter into the model, we therefore restrict our attention to the case $K \equiv 0$, noting that understanding the diffusion term as an eddy diffusion may still be a useful physical interpretation.

\subsubsection{Modification to the unsteady similarity solutions}

In SCDH attracting power-law similarity solutions were found for (2.1). These solutions were observed experimentally in Scase et al. (2008). Again, the validity of the velocity diffusion term $(\varepsilon \neq 0)$ is drawn into question if these similarity solutions are found to be significantly affected by its inclusion. For plumes propagating in an unstratified ambient these similarity solutions are

$$
\hat{Q}=\frac{2 \alpha^{2} \rho_{\infty}}{9} \frac{z^{3}}{t}, \quad \hat{M}=\frac{\alpha^{2} \rho_{\infty}}{9} \frac{z^{4}}{t^{2}}, \quad \hat{F}=\frac{\alpha^{2} \rho_{\infty}}{9} \frac{z^{4}}{t^{3}}\left(1-\frac{\varepsilon}{3}\right)
$$

and these solutions also apply under translations in $z$ and $t$. For such solutions we note that $b=2 \alpha z / 3$ and, as with the steady case, the diffusion term does not enter. Hence, we see that the inclusion of the diffusion term does not affect the predicted narrow radius in the matching region investigated and observed experimentally in Scase et al. (2008). 


\section{Numerical results for top-hat plumes}

The regularized problem is now (2.1) with $(2.1 b)$ replaced by (2.14). To approach this modified problem numerically it is convenient to transform the system. We introduce the variables $A=\rho_{\infty} b^{2}=Q / w$, proportional to the plume's cross-sectional area, and $B=\rho_{\infty} b^{2} g^{\prime}$, proportional to the total buoyancy (NB some authors use $B$ to denote the buoyancy flux which is denoted $F$ herein). We define the vector $\boldsymbol{A}=(A, w, B)^{\top}$ and rewrite the governing model (that is, (2.1) with (2.1b) replaced by (2.14)) as

$$
\begin{gathered}
\frac{\partial A}{\partial t}+\frac{\partial}{\partial z}(w A)=2 \gamma w A^{1 / 2} \\
\frac{\partial w}{\partial t}+\frac{\partial}{\partial z}\left(\frac{1}{2} w^{2}\right)=\frac{B}{A}-2 \gamma \frac{w^{2}}{A^{1 / 2}}+\frac{\varepsilon}{2 \gamma} \frac{\partial}{\partial z}\left(w A^{1 / 2} \frac{\partial w}{\partial z}\right), \\
\frac{\partial B}{\partial t}+\frac{\partial}{\partial z}(w B)=0,
\end{gathered}
$$

where $\gamma \equiv \alpha \rho_{\infty}^{1 / 2}$. In all of the numerical results presented below we take $\gamma=1$, which can be assumed without any loss of generality as it corresponds to a rescaling.

For simplicity we will choose finite source conditions by considering the truncated domain $z \in\left[1, z_{\max }\right]$. At $z=1$ we impose conditions that are consistent with the steady solutions (2.15),

$$
F(z=1, t)=F_{0}(t), \quad Q(z=1, t)=c_{\hat{Q}} F_{0}(t)^{1 / 3}, \quad w(z=1, t)=c_{\hat{w}} F_{0}(t)^{1 / 3},
$$

where $F_{0}(t)$ is a known source function. Owing to the increased order associated with the diffusive term an additional downstream condition is needed, which we choose to be

$$
\frac{z_{\max } w_{z}\left(z=z_{\max }, t\right)}{w\left(z=z_{\max }, t\right)}=-\frac{1}{3} .
$$

This choice is ad-hoc, but it is motivated by the algebraic form of the solution (2.15), which certainly must apply for at least some time after the change in source buoyancy flux. In the results presented below, the exact nature of the boundary condition chosen at $z=z_{\max }$ was not found to significantly affect the solutions obtained.

\subsection{Linearized harmonic perturbations}

We begin by repeating the analysis of $\S 2.1$ but applied to the modified model equations (3.1). We therefore suppose that the source is driven by a buoyancy flux in the form of $F_{0}(t)=1+\delta \mathrm{e}^{\mathrm{i} \omega t}$, where $\delta \ll 1$, and examine the resulting harmonic perturbation $\tilde{\boldsymbol{a}}=(\tilde{A}, \tilde{w}, \tilde{B})^{\top}$, defined analogously to (2.3), where $\boldsymbol{A}_{B}=$ $\left(\hat{Q}_{B} / \hat{w}_{B}, \hat{w}_{B}, \hat{F}_{B} / \hat{w}_{B}\right)^{\top}$, is the steady solution to the modified model, as given by (2.15) and $(2.16 a)$. As before we consider a single Fourier mode and define $\tilde{\boldsymbol{a}}=\tilde{\boldsymbol{A}}(z) \mathrm{e}^{\mathrm{i} \omega t}$.

To solve for the perturbation, $\tilde{A}(z)$, we first linearize (3.1)-(3.2), then discretize using a second-order finite-difference scheme. The resulting banded matrix problem is solved by LU decomposition. The results discussed below are independent of the choice of domain size and spatial resolution.

In figure 3 we show the spatial variation of these linearized perturbations, $\tilde{A}(z)$, for $\omega=2,10$ and a range of the diffusivity parameter $\varepsilon$. In each figure we provide the corresponding solutions for the original model equations with $\varepsilon=0$, as described in $\S 2.1$. We note again that, when $\varepsilon=0$, the harmonic perturbations grow increasingly 
(a)

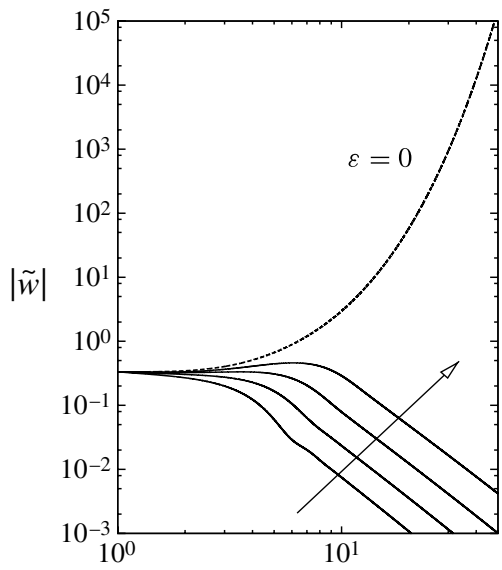

(c)

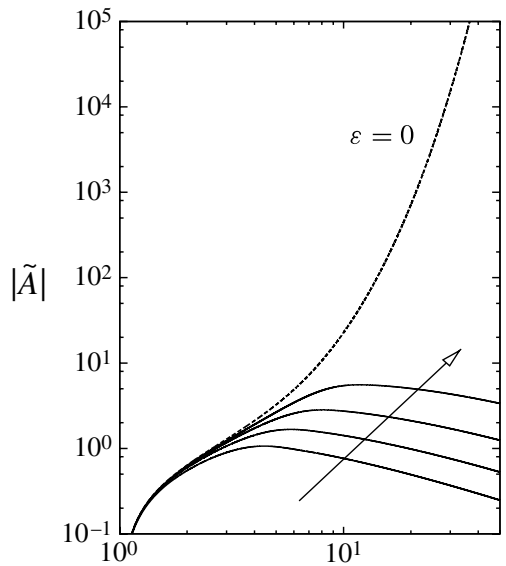

(e)

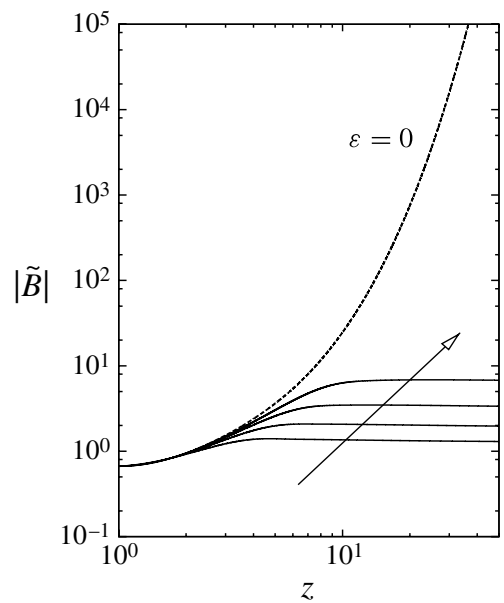

(b)

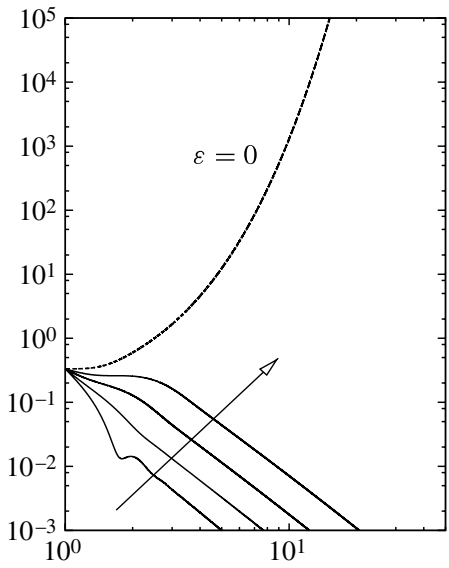

(d)

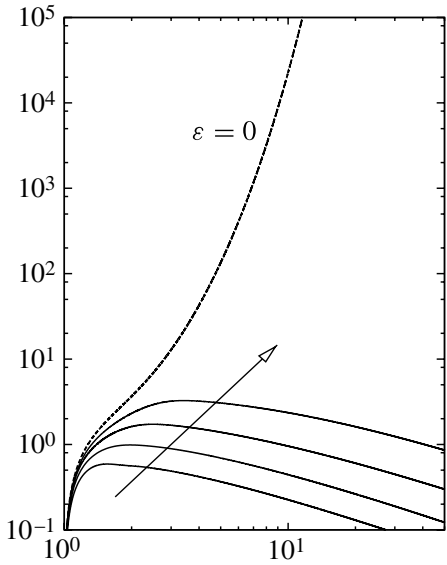

$(f)$

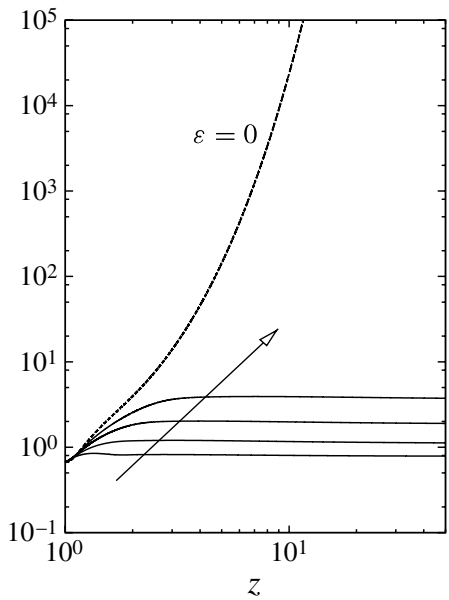

FIGURE 3. The downstream evolution of a linear harmonic perturbation to the modified plume model. The perturbations are oscillatory in $z$ but here we present the absolute value for clarity. The original non-diffusive $(\varepsilon=0)$ model prediction (as discussed in $\S 2.1$ ) is shown as the dashed upper line in each figure, whilst the arrow shows decreasing values of $\varepsilon=0.1,0.05,0.025,0.0125$. $(a, c, e) \omega=2$ and $(b, d, f) \omega=10$. 
quickly as the frequency $\omega$ is increased, as can be observed by a comparison of the left-hand column $(\omega=2)$ and the right-hand column $(\omega=10)$. Such behaviour is not observed in the regularized system at finite values of $\varepsilon$, which renders the initial-value problem well-posed.

In order to estimate an appropriate value for $\varepsilon$ we have considered the laboratory data, from nominally steady-source plumes, published by both Rouse, Yih \& Humphreys (1952) and Papanicolaou \& List (1988). There is inevitably scatter between the data sets, but certainly the values of $\varepsilon$ that we have used in our numerical solutions, specifically $\varepsilon \in(0,0.1]$, are within the range of published experimental data.

\subsection{Nonlinear unsteady initial-value computations}

To solve (3.1) we employ a segregated solver for the unknowns $A_{i}^{(n)}, w_{i}^{(n)}$ and $B_{i}^{(n)}$, over a distribution of nodal points $z_{i}\left(i=1, \ldots, N\right.$, where $z_{1}=1$ and $\left.z_{N}=z_{\max }\right)$ and time points $t_{n}$. At each time level, $t_{n}$, the advection-diffusion equation $(3.1 b)$ is evaluated at the mid-point in time $\left(t_{n}+t_{n-1}\right) / 2$ using a second-order finite-difference scheme. During this solution phase we treat the quantities $A_{i}^{(n)}$ and $B_{i}^{(n)}$ as being known. The advection equations (3.1a) and (3.1c) are solved by a conservative MUSCL approach ('monotone upwind-centred scheme for conservation laws'); this is a finite-volume scheme that uses a piecewise linear approximation in each volume element and is second-order in space and time. For this advection phase we treat $w_{i}^{(n)}$ as being known. The coupled nature of the system is finally taken into account by an iteration for each time step, repeating the above procedure until a tolerance (typically $10^{-8}$ ) is achieved for the maximum change of all discrete variables across the whole spatial mesh at that time step.

The unsteady problem (3.1)-(3.2) requires an initial condition, which we take to be the steady solution (2.15). Therefore, if $F_{0}(t)$ takes a constant value for $t \geqslant 0$, then no unsteady behaviour will be observed in the initial-value problem.

We consider the evolution of the plume following a change in the source conditions. For the source buoyancy flux we choose a rapidly varying (but smooth) profile in the form

$$
F_{0}(t)=F_{i}+\left(F_{f}-F_{i}\right)\left(1-\mathrm{e}^{-100 t^{2}}\right),
$$

which transitions from an initial value $F_{i}$ to a final value of $F_{f}$.

In figure $4(a)$ we show the evolution of the plume radius for a 'turned-down' source with $F_{i}=1, F_{f}=0.01$, including diffusive effects with $\varepsilon=0.05$. Figure $4(b)$ shows the analogous 'turned-up' case with $F_{i}=0.01, F_{f}=1$ and $\varepsilon=0.05$. In both cases, but particularly for increases in source buoyancy flux, the profiles of $A$ and $B$ develop steep gradients. The accurate resolution of these rapid transition regions was the primary motivation for our separate numerical treatment of (3.1a) and (3.1c) via the MUSCL approach.

In figures $4(c)$ and $4(d)$ the influence of varying $\varepsilon \in[0.025,0.1]$ is investigated, again showing the plume radius, but at a fixed time. As $\varepsilon \rightarrow 0$, a 'spike' develops that is associated with an increase in plume radius; this feature is clear for the turned-up case, but it is also present for turned-down evolutions, as highlighted by the circle in figure $4(c)$. At finite values of $\varepsilon$ the same spike develops with time, as is most clearly seen in figure 4(b). In figure $4(c)$ we also show a (dashed) line segment with a $2 / 3$ gradient, which is a prediction for the neck region flow given by SCDH (for $\varepsilon=0$ ).

Physically, the reason for the formation of the spike at the bottom of the neck in the turned-down realizations is the different power-law behaviour of the plume's 
(a)

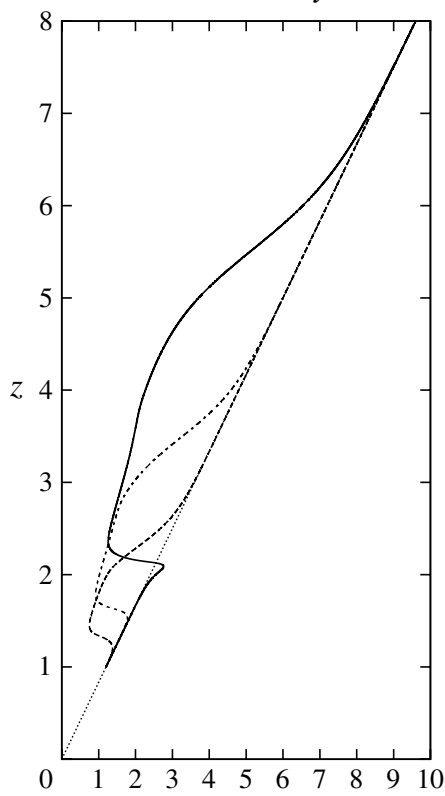

(c)

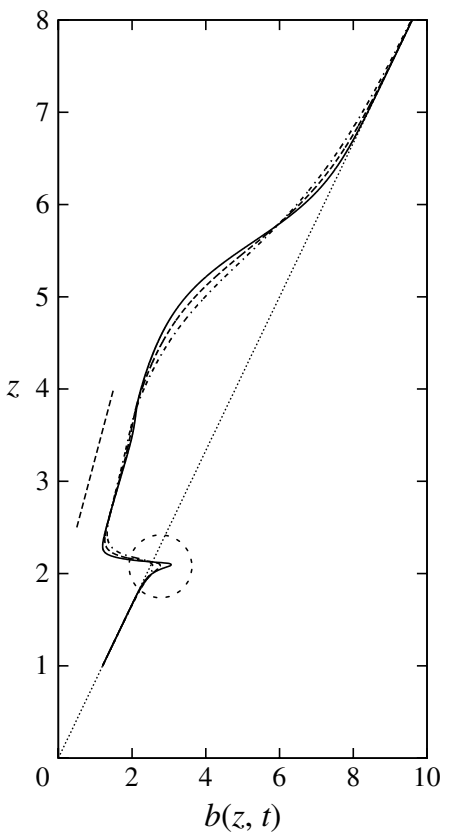

(b)

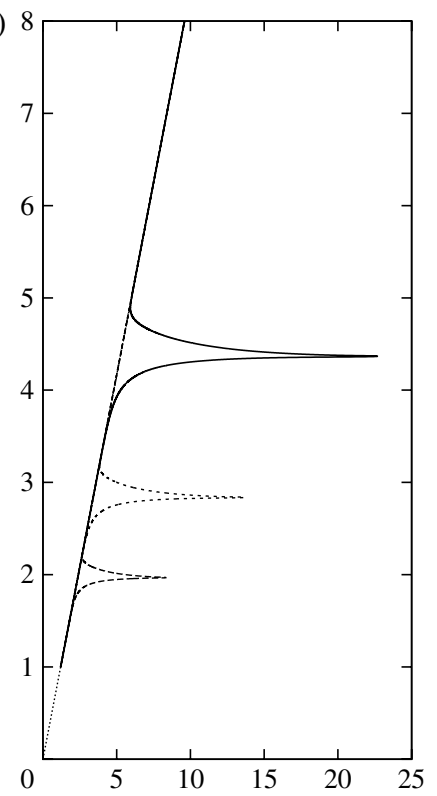

(d)

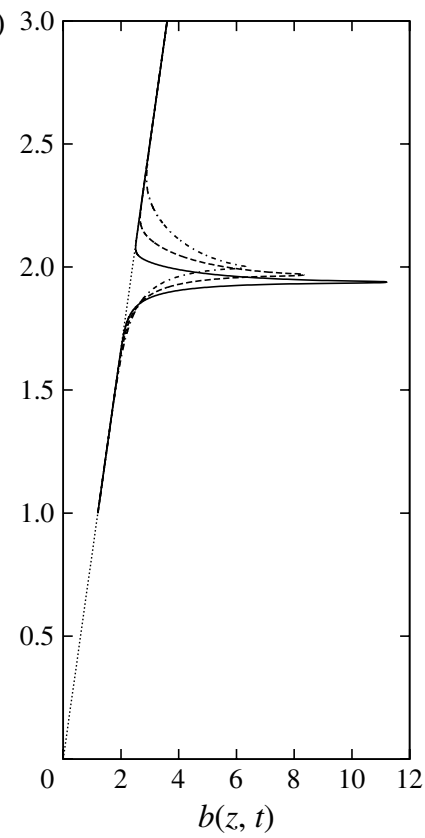

FIGURE 4. The Boussinesq plume radius $b=\sqrt{A}$ for a range of evolutions using the source profile (3.3) with (a) $F_{i}=1, F_{f}=0.01, \varepsilon=0.05, t=2(---), 4(--), 8(-)$; (b) $F_{i}=0.01$, $F_{f}=1, \varepsilon=0.05, t=2(---), 4(--), 8(-) ;(c) F_{i}=1, F_{f}=0.01, t=8, \varepsilon=0.1(---)$, $0.05(-\cdot-\cdot), 0.025(-) ;(d) F_{i}=0.01, F_{f}=1, t=2, \varepsilon=0.1(---), 0.05(-\cdot-\cdot), 0.025(-)$. The (dotted) straight line shows the solution for a steady plume radius in each case.

velocity in the necked (above) and lower (below) regions. Below the neck the plume is following the classical $w \sim z^{-1 / 3}$ law and fluid just below the neck is therefore rising as $z \sim t^{3 / 4}$. In the neck, the velocity is behaving as $w \sim z / 2 t$ and so fluid at the base of 
(a)

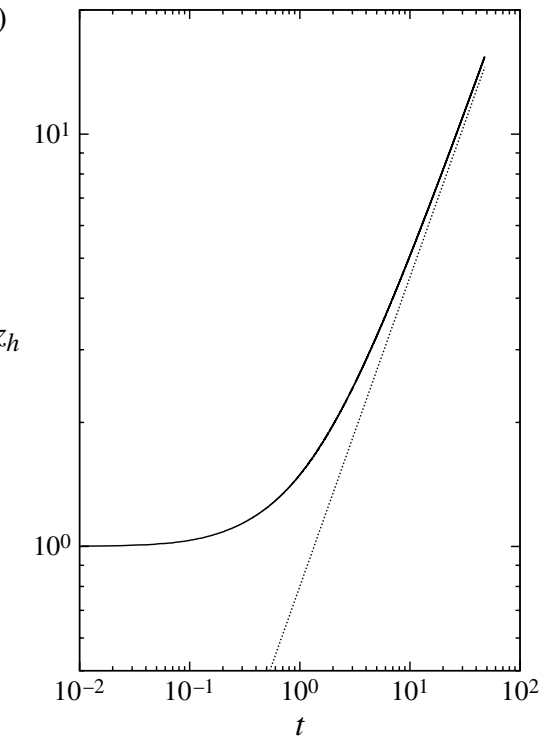

(b)

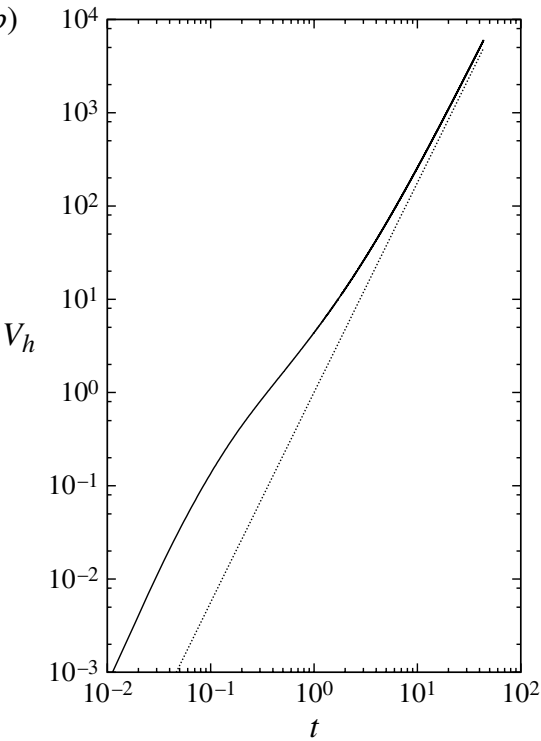

FIGURE 5. For the turned-up finite-source computation presented in figure 4(b), we compute: (a) the height of the spike in plume radius, $z_{h}$, and $(b)$ the additional volume of the spike $V_{h}$ as defined by (3.4). The gradient of the dotted lines in each figure are the predictions of Scase et al. (2009): (a) $z_{h} \sim t^{3 / 4}$; and (b) $V_{h} \sim t^{9 / 4}$.

the neck is rising as $z \sim t^{1 / 2}$. Thus, as time increases, fluid at the bottom of the neck is swept up by fluid at the top of the lower plume, causing the formation of the spike.

The formation of the spike in the turned-up case is perhaps more intuitive despite the same $z^{-1 / 3}$ power-law behaviour of the velocity field either side of the spike. The spike forms because the plume is adjusting to the faster source conditions, and hence faster fluid is moving into regions of slower, less buoyant, fluid not withstanding the usual $z^{-1 / 3}$ decay in velocity.

In Scase et al. (2009) various scaling laws were suggested for the behaviour of the spike, in the absence of explicit momentum diffusion. It was argued that the vertical position of the spike scaled as $z_{h} \sim t^{3 / 4}$, and the volume of fluid contained within the spike scaled as $V_{h} \sim t^{9 / 4}$. These scalings were derived for point-source, pure plumes. It was proposed that plumes emanating from finite-sized sources tend toward following these scalings far from the source. In figure 5 we present data for $z_{h}$ and

$$
V_{h}(t)=\pi \int_{z=1}^{z=z \max }\left\{A(z, t)-\left(\frac{6 z}{5}\right)^{2}\right\} \mathrm{d} z ;
$$

in each figure the dotted lines show the predicted large-time $t^{3 / 4}$ and $t^{9 / 4}$ gradients.

\section{Unsteady Gaussian plume models}

The modelling of turbulent plumes under an assumption of Gaussian self-similarity is popular as the model fits experimental data more closely than an assumed top-hat profile. However, the modelling of the unsteady behaviour of a plume with an assumed Gaussian profile presents additional difficulties compared to modelling plumes with an assumed top-hat profile. In particular, replicating the analysis presented in SCDH fails for 'Gaussian plumes' as the integral of the mass of the plume at a given height does 
not converge. Specifically,

$$
\int_{0}^{\infty} \rho r \mathrm{~d} r
$$

does not exist (cf. the first term of (2.4) in SCDH and the first term of (1) in Yu 1990). The quantity (4.1) arises due to the inclusion of the unsteady part of the convective derivative in the mass conservation equation. A top-hat plume has finite mass in its cross-section, conversely a Gaussian plume has an infinite mass in its cross-section and so lacks the compact support necessary to make the analysis of SCDH work. In a steady framework, arguments can be made about entrainment into a plume with Gaussian profiles, and indeed where this entrainment into the plume occurs, since the plume has no edge (see e.g. Scase et al. 2007). However, when integral properties of the plume are to be tracked, integrating over the plume cross-section fails in the Gaussian case (4.1) as a result of this infinite mass. A naïve approach to circumvent the problem would be to attempt to subtract the steady ambient background density, $\rho_{\infty}$, from the plume density, $\rho$ (both multiplied by the radial ordinate), since it is only the time derivative that is required. This attempt leads only to the density deficiency, or buoyancy flux, equation though.

Despite this lack of convergence, several authors have presented models for unsteady Gaussian plume behaviour. Here we consider the three different models posed by Delichatsios (1979), Yu (1990) and Vul'fson \& Borodin (2001) (see also Vul'fson $2001 a, b)$.

To introduce the models of Delichatsios (1979) and Yu (1990), we assume that the plume has Gaussian profiles in the radial direction, specifically

$$
w(r, z)=w_{0}(z) \exp \left\{-r^{2} / b^{2}\right\}, \quad g^{\prime}(r, z)=g_{0}^{\prime}(z) \exp \left\{-r^{2} /\left(\lambda^{2} b^{2}\right)\right\} .
$$

Here, the ratio of temperature radius to velocity radius is $\lambda$. The governing equations for unsteady Gaussian plumes due to both Delichatsios (1979) and Yu (1990) can be represented by

$$
\begin{gathered}
\kappa \frac{\partial}{\partial t}\left(b^{2}\right)+\frac{\partial}{\partial z}\left(b^{2} w_{0}\right)=2 \alpha b w_{0}, \\
\frac{\partial}{\partial t}\left(b^{2} w_{0}\right)+\frac{1}{2} \frac{\partial}{\partial z}\left(b^{2} w_{0}^{2}\right)=\lambda^{2} b^{2} g_{0}^{\prime}, \\
\frac{\partial}{\partial t}\left(b^{2} g_{0}^{\prime}\right)+\frac{1}{1+\lambda^{2}} \frac{\partial}{\partial z}\left(b^{2} w_{0} g_{0}^{\prime}\right)=0,
\end{gathered}
$$

where $\kappa=1$ corresponds to the model of Delichatsios (1979) and $\kappa=2$ is the model of $\mathrm{Yu}$ (1990). The details of the derivation of the model due to Delichatsios (1979) are brief, stating that the model is the appropriate unsteady generalization of mass, momentum and energy conservation. Further exposition is given in Yu (1990), although as discussed above the attempt to use a divergent integral renders the model's origins doubtful. The model of Vul'fson \& Borodin (2001) has identical equations for conservation of momentum and buoyancy as Delichatsios (1979) and Yu (1990), but avoids the problems associated with the conservation of mass by replacing (4.3a) with the assumption that the plume is conical at all times. This assumption that $b(z, t) \sim z$ is very strong, but is based on similar assumptions by Schmidt (1941), Prandtl (1952) and Schmidt (1957) in steady contexts. It is an open question as to how appropriate such a strong assumption is in an unsteady context. In the special case $\lambda=1$ the model of $\mathrm{Yu}((4.3)$, with $\kappa=2)$ can be scaled such that it is identical to the top-hat 
model of SCDH (2.1) and so the dynamical properties, and in particular the response to perturbations at the source, of the two models are similar in this case.

We note here that the Gaussian models (4.3) accept power-law solutions in the form

$$
b=\frac{2 \alpha z}{3}, \quad w=\frac{1+\lambda^{2} z}{2} \frac{z}{t}, \quad g^{\prime}=\frac{1+\lambda^{2}}{2} \frac{z}{t^{2}} .
$$

As with the top-hat model, $w$ and $g^{\prime}$ are independent of the entrainment coefficient, $\alpha$.

\subsection{Difficulties associated with unsteady Gaussian plume models}

We can repeat the analysis of $\S 2$ for the Gaussian plume models, and similarly determine if those models are well-posed or not. In each case, the non-dimensional quantities $\zeta$ and $\tau$ are defined as $\zeta=\left(z \omega / w_{B}\right)^{1 / 2}$, and $\tau=\omega t$, for the appropriate steady basic flow velocity $w_{B}$. In the case that $\lambda=1$, the model of Yu (1990) predicts exactly the same perturbation quantities $\boldsymbol{q}$ as the non-regularized, $\varepsilon=0$, top-hat model, due to the fact that the two models differ only by a scaling. However, the model of Delichatsios (1979) produces significantly more unstable oscillations with (for $\lambda=1$ )

$$
\begin{aligned}
f= & \frac{3 \mathrm{i}}{4 \zeta^{5 / 4}} \mathrm{e}^{\mathrm{i}\left[\tau-3 / 4 \zeta^{2}\right]}\left\{\mathscr{I}_{7 / 8}\left(\frac{3 \zeta^{2}}{4}\right) \int_{0}^{\zeta}\left(\mathrm{i}-2 \xi^{2}\right) \xi^{1 / 4} \mathrm{e}^{-3 \mathrm{i} / 4 \xi^{2}} \mathscr{K}_{7 / 8}\left(\frac{3 \xi^{2}}{4}\right) \mathrm{d} \xi\right. \\
& \left.-\mathscr{K}_{7 / 8}\left(\frac{3 \zeta^{2}}{4}\right) \int_{0}^{\zeta}\left(\mathrm{i}-2 \xi^{2}\right) \xi^{1 / 4} \mathrm{e}^{-3 \mathrm{i} / 4 \xi^{2}} \mathscr{I}_{7 / 8}\left(\frac{3 \xi^{2}}{4}\right) \mathrm{d} \xi\right\},
\end{aligned}
$$

where $\mathscr{I}$ and $\mathscr{K}$ are modified Bessel functions of the first and second kind; whereas, the model of Vul'fson \& Borodin (2001) produces more physically realistic solutions:

$$
f=\left(\frac{4}{3 \lambda^{2}}\right)^{3 / 4} \frac{1}{\zeta^{3 / 2}} \exp \left\{\mathrm{i} \tau-\frac{3 \mathrm{i}}{8}\left[\pi+\zeta^{2}\left(2+\lambda^{2}\right)\right]\right\} \mathscr{M}_{\left(\lambda^{2}-2\right) / 4 \lambda^{2}, 1 / 4}\left(\frac{3}{4} \mathrm{i} \lambda^{2} \zeta^{2}\right)
$$

where $\mathscr{M}$ is the Whittaker-M function (see e.g. Abramowitz \& Stegun 1965).

Figure 6 shows representative growths of the oscillations for the four models under consideration (the non-regularized, $\varepsilon=0$, top-hat model and the model of Yu 1990 coincide). The model of Vul'fson \& Borodin (2001) has a benign response, with the oscillations decaying away with height; however the extremely strong restriction that $b=6 \alpha z / 5$ (for all time) makes the model unattractive if larger perturbations are considered.

The analysis can be repeated under the assumption that $F \equiv 0$, and the systems are modelling the evolution of unsteady turbulent jets. The same qualitative results are found, and are shown in the Appendix.

\subsection{Regularization and numerical solution of the Gaussian plume models}

The Gaussian models, as described by (4.3) may also be cast into a form that is analogous to (3.1). By including momentum diffusion (as before, with coefficient $\varepsilon$ ) we obtain the modified model:

$$
\begin{gathered}
\kappa \frac{\partial A}{\partial t}+\frac{\partial}{\partial z}\left(w_{0} A\right)=2 w_{0} A^{1 / 2} \\
\frac{\partial w_{0}}{\partial t}+\left(1-\frac{1}{\kappa}\right) \frac{\partial}{\partial z}\left(\frac{1}{2} w_{0}^{2}\right)=\lambda^{2} \frac{B}{A}-2 \frac{w_{0}^{2}}{\kappa A^{1 / 2}} \\
+\left(\frac{1}{\kappa}-\frac{1}{2}\right) \frac{w_{0}^{2}}{A} \frac{\partial A}{\partial z}+\frac{\varepsilon}{2} \frac{\partial}{\partial z}\left(w_{0} A^{1 / 2} \frac{\partial w_{0}}{\partial z}\right),
\end{gathered}
$$


(a)

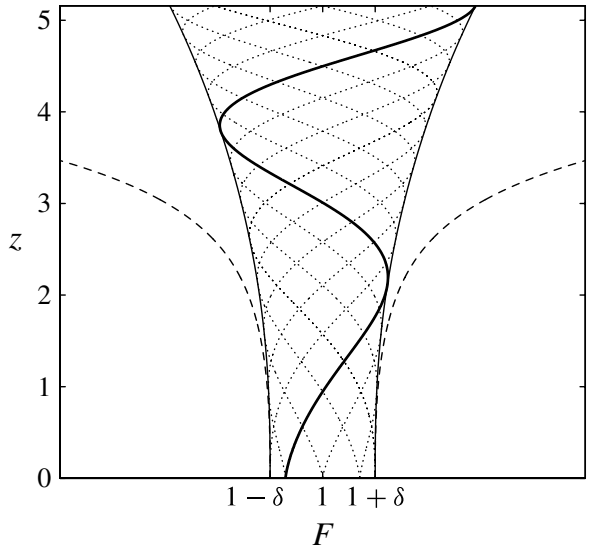

(b)

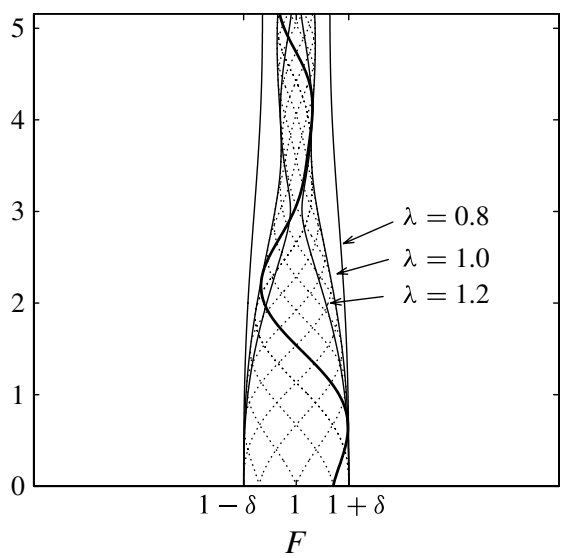

FIGURE 6. The growth of oscillations in the buoyancy flux field. (a) The top-hat model: the thick solid line shows the field at a representative time. The thin solid boundary shows the envelope of $F$ and hence the growth of the perturbation. The outer dashed line is the envelope for the model due to Delichatsios (1979). (b) The decay of oscillations in the Vul'fson \& Borodin (2001) model for $\lambda=1$. The envelopes for differing values of $\lambda$ are also shown.

$$
\frac{\partial B}{\partial t}+\frac{1}{1+\lambda^{2}} \frac{\partial}{\partial z}\left(w_{0} B\right)=0,
$$

where, without loss of any generality, we have set the entrainment coefficient, $\alpha$, to be unity. Here we have kept a notation that is consistent with (3.1), using $A=b^{2}$, $B=b^{2} g_{0}^{\prime}$. We must also note that the steady solutions to this system are slightly modified from the top-hat case, in particular one must replace $c_{Q} \rightarrow c_{Q}\left(2 \lambda^{2}\right)^{1 / 3}$ in (2.2).

For $\kappa=2$, Yu (1990), we have already noted that this system (when $\lambda=1$ ) is equivalent to the top-hat model of SCDH after a suitable rescaling. Therefore, our previous discussion regarding the solution and regularization of the top-hat model also applies to this system.

In the case $\kappa=1$, Delichatsios (1979), we have investigated the response to linear harmonic perturbations as per the discussion $\S 3.1$. The growth of small-scale waves is much more severe in this model when $\varepsilon=0$ and our investigation for $\varepsilon \neq 0$ reveals that the system remains ill-posed even in the presence of velocity diffusion. In this sense we feel that this model is ill-suited to modelling such flows.

In figure 7 we present results at fixed time for the evolution of a turned-up and turned-down source for a range of values of $\lambda=0.5,1$ and 1.5. These computations are developed using the same scheme as described in $\S 3$, starting from a steady-state solution with the same transition profile (3.3).

\section{Similarity solutions}

Similarity solutions to unsteady integral plume models have been considered by previous authors. It has been found experimentally that the source conditions of a plume generated by a fast-growing fire are well-approximated by power-law representations. As a result, similarity solutions can be used to predict detector, or collector, measurement response, see for example Delichatsios (1979) and Yu (1990). In the present section we revisit the previously derived similarity solutions and demonstrate that for the range of power-law solutions considered in the literature 
(a)

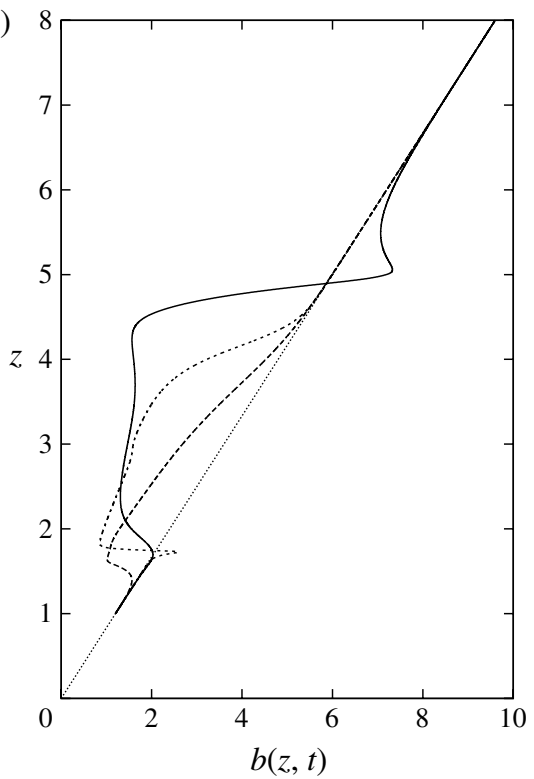

(b)

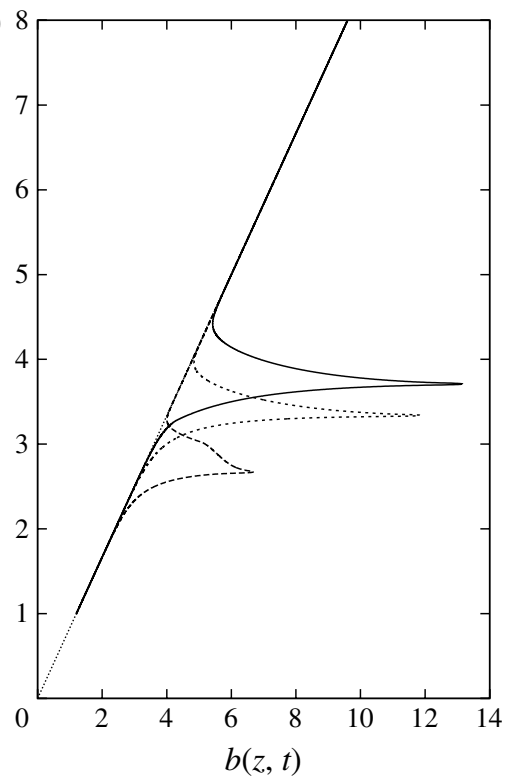

FIGURE 7. The plume radius $b(z, t)$ in the modified Gaussian model $(\kappa=2)$ for $(a) F_{i}=1$, $F_{f}=0.01, \varepsilon=0.05, t=8$ and $(b) F_{i}=0.01, F_{f}=1, t=8, \varepsilon=0.05$. In both cases $\lambda=0.5$ $(---), 1(--)$ and $1.5(-)$. The dotted line shows the solution for a steady plume radius.

there is very little difference in the model predictions. We include predictions with and without diffusion and show that, again, regularizing the models does not significantly affect predictions that have previously been empirically validated; we discuss this validation further below.

\subsection{Governing equations}

Following the methodology of Delichatsios (1979) and Yu (1990), we define a similarity variable $\eta=z /\left(\Gamma t^{n}\right)$, and rescale the primary variables according to

$$
b=\tilde{b} \Gamma t^{n}, \quad w=\tilde{w} \Gamma n t^{n-1}, \quad g^{\prime}=\tilde{g}^{\prime} \Gamma n(n-1) t^{n-2},
$$

for $n \neq 0,1$. We seek solutions for $\left.b^{2} w g^{\prime}\right|_{z=0} \propto t^{p}$ (and take the constant of proportionality to be $c$ ). To attain similarity solutions it follows that $p=4 n-3$ as shown by Delichatsios (1979) and Yu (1990). Making the substitution (5.1) into (4.3) we find

$$
\begin{gathered}
\kappa\left\{2 \tilde{b}^{2}-\eta \frac{\mathrm{d} \tilde{b}^{2}}{\mathrm{~d} \eta}\right\}+\frac{\mathrm{d}}{\mathrm{d} \eta}\left(\tilde{b}^{2} \tilde{w}\right)-2 \alpha \tilde{b} \tilde{w}=0, \\
\left\{(3 n-1) \tilde{b}^{2} \tilde{w}-n \eta \frac{\mathrm{d}}{\mathrm{d} \eta}\left(\tilde{b}^{2} \tilde{w}\right)\right\}+\frac{n}{2} \frac{\mathrm{d}}{\mathrm{d} \eta}\left(\tilde{b}^{2} \tilde{w}^{2}\right)-\lambda^{2}(n-1) \tilde{b}^{2} \tilde{g}^{\prime}=0, \\
\left\{\frac{(3 n-2)}{n} \tilde{b}^{2} \tilde{g}^{\prime}-\eta \frac{\mathrm{d}}{\mathrm{d} \eta}\left(\tilde{b}^{2} \tilde{g}^{\prime}\right)\right\}+\frac{1}{1+\lambda^{2}} \frac{\mathrm{d}}{\mathrm{d} \eta}\left(\tilde{b}^{2} \tilde{w} \tilde{g}^{\prime}\right)=0
\end{gathered}
$$

where the quantities in curly brackets are due to the time derivatives in (4.3). Solutions to the steady system, where the quantities in curly brackets are ignored, are first sought. They are identical for both the Delichatsios (1979) and Yu (1990) systems and 


$\begin{array}{ll}\text { Note } & \text { Comment } \\ \text { I } & \text { The factor of } 2 \text { is replaced with } 1 \text { for the Delichatsios (1979) model. } \\ \text { II } & \text { A typographical error in Yu (1990) replaces this with a 1. } \\ \text { III } & \text { The term is omitted in the Delichatsios (1979) model. } \\ \text { IV, VII } & \text { Identically zero in the Vul'fson \& Borodin (2001) model. } \\ \text { V } & \text { The factor is only present in the Vul'fson \& Borodin (2001) model. } \\ \text { VI } & \text { Becomes } 2 \bar{w}^{2} \text { in the Vul'fson \& Borodin }(2001) \text { model since } \bar{b}=1 . \\ (5.6 a) & \text { Is replaced by } \bar{b}=1 \text { in the Vul'fson \& Borodin }(2001) \text { model. } \\ (5.6 c) & \text { The top-hat model replaces the factor } 2\left(1+\lambda^{2}\right)^{-1} \text { with } 1 \text { throughout. }\end{array}$

TABLE 1. The required adjustments to the Yu (1990) model, (5.6), in order to apply the models of Delichatsios (1979) and Vul'fson \& Borodin (2001) and the top-hat SCDH model.

are given by

$$
\begin{aligned}
& \tilde{b}_{u}=\frac{6 \alpha}{5} \eta, \quad \tilde{w}_{u}=\frac{5}{3}\left(\frac{9}{20}\right)^{1 / 3}\left(\frac{2 \alpha \rho_{\infty}^{1 / 2}}{F_{0}^{1 / 2}}\right)^{-2 / 3}\left(\frac{2 c \lambda^{2}}{n^{3}}\right)^{1 / 3} \eta^{-1 / 3}, \\
& \tilde{g}_{u}^{\prime}=\frac{5}{3}\left(\frac{9}{20}\right)^{-1 / 3}\left(\frac{2 \alpha \rho_{\infty}^{1 / 2}}{F_{0}^{1 / 2}}\right)^{-4 / 3}\left(\frac{2 c \lambda^{2}}{n^{3}}\right)^{2 / 3} \frac{n}{2 \lambda^{2}(n-1)} \eta^{-5 / 3}
\end{aligned}
$$

for $\Gamma=\left(F_{0} / \rho_{\infty}\right)^{-1 / 4}$. We now define perturbation quantities such that

$$
\bar{b}=\tilde{b} / \tilde{b}_{u}, \quad \bar{w}=\tilde{w} / \tilde{w}_{u}, \quad \bar{g}^{\prime}=\tilde{g}^{\prime} / \tilde{g}_{u}^{\prime},
$$

and finally rescale by taking

$$
\bar{z}=\frac{p}{n} \eta^{4 / 3}\left[\frac{5}{3}\left(\frac{9}{20}\right)^{1 / 3}\left(\frac{2 \alpha \rho_{\infty}^{1 / 2}}{F_{0}^{1 / 2}}\right)^{-2 / 3}\left(\frac{2 c \lambda^{2}}{n^{3}}\right)^{1 / 3}\right]^{-1}
$$

For all four models, following the above procedure leads to

$$
\begin{gathered}
8 \bar{z}[\bar{w}-\underbrace{2}_{\mathrm{I}} \frac{n \bar{z}}{p}] \frac{\mathrm{d} \bar{b}}{\mathrm{~d} \bar{z}}+4 \bar{z} \bar{b} \frac{\mathrm{d} \bar{w}}{\mathrm{~d} \bar{z}}=5 \bar{w}(1-\bar{b}), \\
4 \bar{z}[\underbrace{\bar{w}}_{\mathrm{II}}-\underbrace{2 \frac{n \bar{z}}{p}}_{\mathrm{III}}] \overline{\bar{w}} \underbrace{\frac{\mathrm{d} \bar{b}}{\mathrm{~d} \bar{z}}}_{\mathrm{IV}}+4 \bar{z}[\frac{n \bar{z}}{p}-\underbrace{\bar{w}}_{\mathrm{V}}] \frac{\mathrm{d} \bar{w}}{\mathrm{~d} \bar{z}}=\underbrace{5 \frac{\bar{w}^{2}}{\bar{b}}-3 \bar{w}^{2}}_{\mathrm{VI}}-2 \bar{g}^{\prime}+\bar{w} \bar{z}, \\
2\left[\frac{2}{1+\lambda^{2}} \bar{w}-2 \frac{n \bar{z}}{p}\right] \underbrace{\frac{\mathrm{d} \bar{b}}{\mathrm{~d} \bar{z}}}_{\mathrm{VII}}+\frac{2}{1+\lambda^{2}} \frac{\mathrm{d} \bar{w}}{\mathrm{~d} \bar{z}}+\frac{1}{\bar{g}^{\prime}}\left[\frac{2}{1+\lambda^{2}} \bar{w}-2 \frac{n \bar{z}}{p}\right] \frac{\mathrm{d} \bar{g}^{\prime}}{\mathrm{d} \bar{z}}=-1,
\end{gathered}
$$

where the meaning of the underbraces, describing the differences between the models, is given in table 1 (cf. (3.4) in Delichatsios 1979, (11)-(13) in Yu 1990). Asymptotic approximations for all four models for small $\bar{z}$ are shown in table 2 (cf. (3.8) in Delichatsios 1979, noting that there is a typographical error in (3.8c)). 

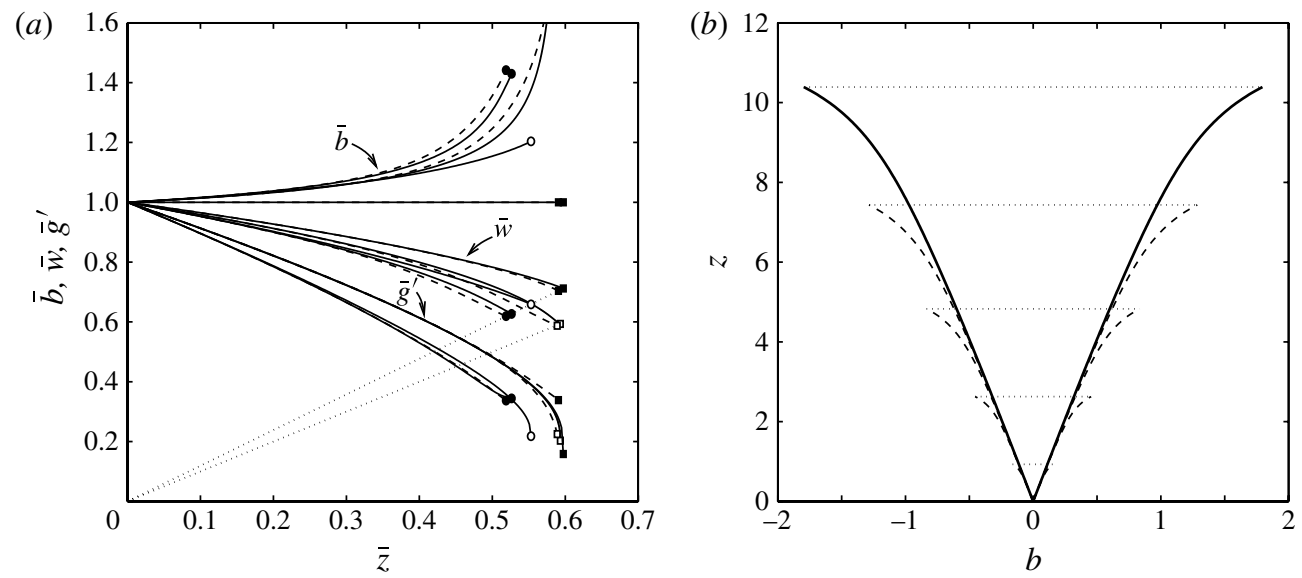

FIgure 8. (a) The similarity solution $\bar{b}, \bar{w}$ and $\bar{g}^{\prime}$ using the models of: ॰, Delichatsios (1979); •, Yu (1990); $\square$, top-hat; and $\mathbf{\square}$, Vul'fson \& Borodin (2001) with (dashed line) and without (solid line) the effects of diffusion $(\varepsilon=0.1)$. The figure shows the solutions for $n=3 / 2$, $p=3$, and $\lambda=0.85^{-1}$ for comparison with figure 1 in $\mathrm{Yu}$ (1990). The upper dotted line is $n\left(1+\lambda^{2}\right) \bar{z} / p$ and the singularity in $(5.6 c)$ occurs at the intersection of this line and $\bar{w}$ except for the top-hat case. In the top-hat case the singularity occurs at the intersection of $\bar{w}$ and $2 n \bar{z} / p$, the lower dotted line. (b) The development of the starting plume under the model of $\mathrm{Yu}$ (1990) with $\lambda=0.85^{-1}, p=3$ and $\varepsilon=0.1$.

\begin{tabular}{|c|c|c|c|}
\hline Quantity & $\begin{array}{c}\text { Yu (1990) and } \\
\text { Delichatsios (1979) }\end{array}$ & Vul'fson \& Borodin (2001) & Top-hat \\
\hline $\bar{b}$ & $1+\frac{2\left(2+\lambda^{2}\right)}{49} \bar{z}+O\left(\bar{z}^{2}\right)$ & 1 & $1+\frac{6}{49} \bar{z}+O\left(\bar{z}^{2}\right)$ \\
\hline $\bar{w}$ & $1-\frac{13\left(2+\lambda^{2}\right)}{98} \bar{z}+O\left(\bar{z}^{2}\right)$ & $1-\frac{2+\lambda^{2}}{10} \bar{z}+O\left(\bar{z}^{2}\right)$ & $1-\frac{39}{98} \bar{z}+O\left(\bar{z}^{2}\right)$ \\
\hline $\bar{g}^{\prime}$ & $1-\frac{39+44 \lambda^{2}}{98} \bar{z}+O\left(\bar{z}^{2}\right)$ & $1-\frac{3+4 \lambda^{2}}{10} \bar{z}+O\left(\bar{z}^{2}\right)$ & $1-\frac{83}{98} \bar{z}+O\left(\bar{z}^{2}\right)$ \\
\hline
\end{tabular}

If, in addition the effects of a velocity diffusion are included in these four models an extra term is required on the right-hand side of $(5.6 b)$, given by

$$
\frac{\varepsilon}{5}\left\{\bar{w}^{2}\left[\bar{z} \frac{\mathrm{d} \bar{b}}{\mathrm{~d} \bar{z}}-\bar{b}\right]-8 \bar{b}^{2} \frac{\mathrm{d}}{\mathrm{d} \bar{z}}\left[\bar{b} \bar{w} \frac{\mathrm{d} \bar{w}}{\mathrm{~d} \bar{z}}\right]+\bar{g}^{\prime}\right\} .
$$

Figure $8(a)$ is a comparison of the similarity solutions of the four models under consideration, with and without diffusion. The figure illustrates that the differences between the four models are small and hence demonstrates that the experimental observations used to verify these models are unlikely to indicate either which model should be preferred or indeed that the underlying models are ill-posed. The model of 
Delichatsios (1979) with diffusion is not shown as the inclusion of diffusion fails to regularize the model. Figure $8(b)$ shows the development of a starting plume under the Yu (1990) model with boundary condition $F_{0}(t)=t^{3}$, for $t \geqslant 0, M_{0}(t)=Q_{0}(t)=0$ with regularizing diffusion $\varepsilon=0.1$. The profile is shown dashed for $t=1,2,3,4$ and solid for $t=5$. The position of the front is indicated at each time by a horizontal dotted line. The position of the front varies as $z_{h} \sim t^{3}$ (and $t^{p}$ in general) unlike the $t^{3 / 4}$ behaviour observed in the bounded turned-up cases considered in $\S 3$.

\subsection{Implications for experimental comparisons}

Here we replot the results of the experiments of Delichatsios (1979) and Yu (1990) and make comparisons between the original ill-posed models (via similarity solutions), and the Yu (1990) and SCDH models regularized by the inclusion of diffusion.

Figure $9(a)$ is a reproduction of figure 2 in Delichatsios (1979) showing the family of similarity solutions for $p=2, \lambda=1.16$. The solid lines are the numerical similarity solution and the dashed line is the first-order asymptotic solution as given in table 2 . Figure $9(b)$ further illustrates this point by comparing experimental data to the model predictions (cf. figure 4 in Delichatsios 1979). The difference between the ill-posed original model and the other models under consideration, with and without diffusion, is small compared to the scatter in the experimental data.

Figure 10 compares the performance of all four models against the experimental data of $\mathrm{Yu}$ (1990) (cf. figures 7 and 8, tests 3 and 4 in Yu 1990). These results were reproduced by Vul'fson \& Borodin (2001) although their plotted model solutions are erroneous. Figure 10(a) illustrates that all the models over-predict the velocity of the plume as may be expected (see the arguments relating to the significance of entrainment into the turbulent cap preceding a starting plume in Scase et al. 2009). The regularized model of $\mathrm{Yu}$ that includes the self-similar Gaussian profiles of velocity and density, of differing radius, is in closest agreement with the experimental data. However, the difference between the four models is comparable with the scatter in the data. Figure $10(b)$ shows that the regularized model of $\mathrm{Yu}$ (1990) is in closest agreement with the data again.

Figures 9 and 10 demonstrate that, like the classical model, the omission of a diffusion term does not significantly affect the predictions of the various models (in the special case of these self-similar solutions). The experimental validation of these models exhibited sufficient scatter in the data that the deficiencies of the models as presented were not realized. Further, validation of the full unsteady models by comparing the similarity solutions with experimental data, from experiments designed to replicate those similarity solutions, was not sufficient to illustrate the ill-posedness of the models.

\section{Conclusions}

We have revisited four existing integral models for describing the behaviour of unsteady plumes and found all four models to be unsuitable in some regard. The model of Vul'fson \& Borodin (2001) is well-defined and well-posed but is bound by the strong assumption that the plume remains conical at all times. The models of SCDH, Yu (1990), and Delichatsios (1979) were all found to be ill-posed, possessing unbounded temporal growth of infinitesimally short waves. In addition, the derivation of the Gaussian models makes use of a divergent integral for the mass in the plume.

The models of SCDH and Yu (1990) are regularized by the inclusion of a velocity diffusion term. This extra term in the model can be considered to mirror the vertical 

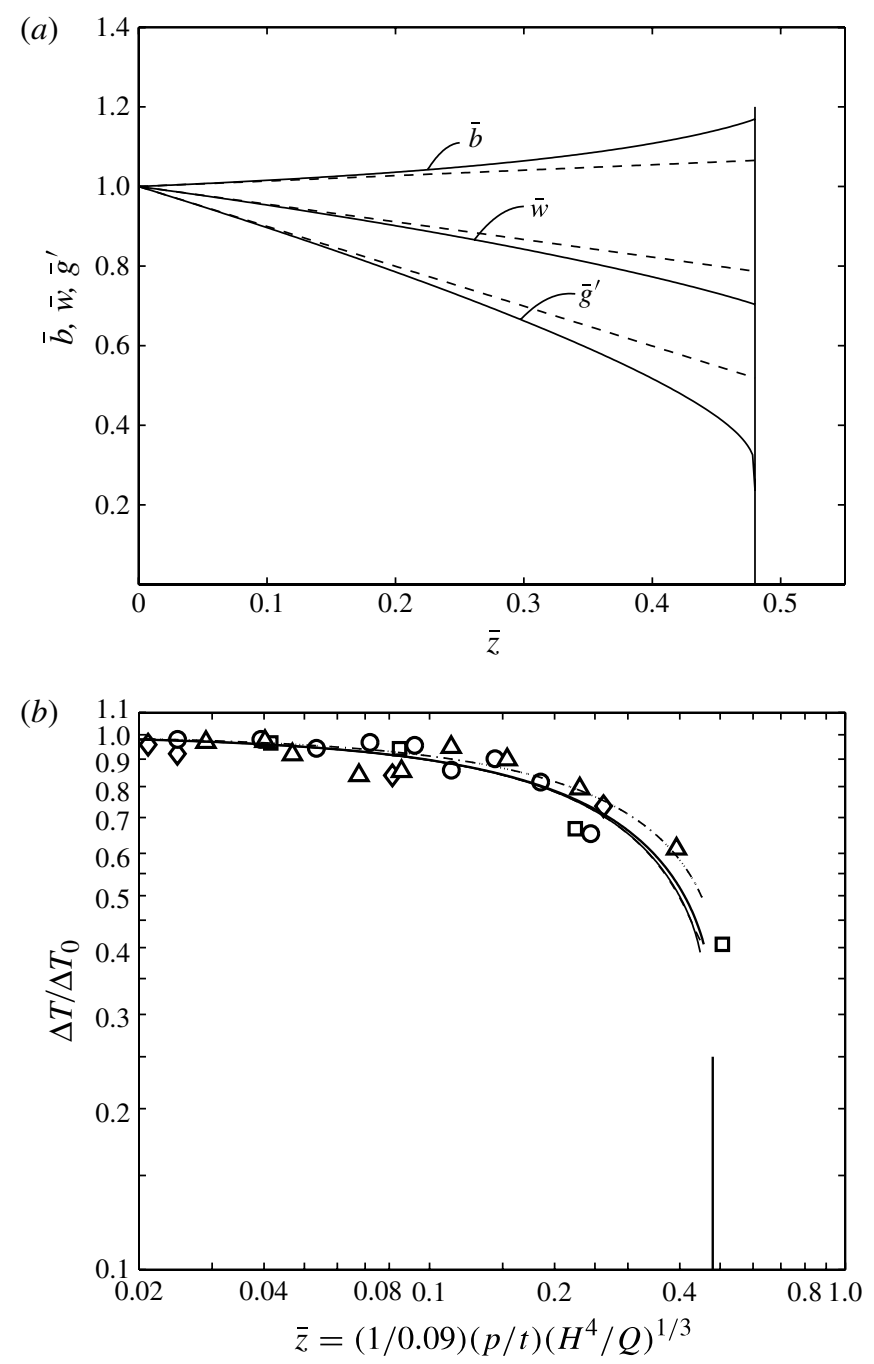

FIGURE 9. (a) Dimensionless radial spread, velocity, and buoyancy of an unsteady plume, expressed as fractions of the quasi-steady values, versus the dimensionless vertical position (cf. figure 2 in Delichatsios 1979): - numerical solution $(\varepsilon=0)$; --- , first-order asymptotic solution, $\lambda=1.16 ; p=2.0$. (b) Comparison of experimental results with the theoretical predictions (cf. figure 4 Delichatsios 1979): temperature histories over ignition expressed as fractions of the quasi-steady values versus time expressed in terms of the dimensionless parameter $\bar{z}$. Early times correspond to large values of $\bar{z}$. ( $t$ in s, $H$ in m, $Q$ in W - here using the notation of Delichatsios 1979.) - Numerical solution for $\varepsilon=0$ under the Delichatsios (1979) model; - - , numerical solution for $\varepsilon=0$ under the Yu (1990) model; - , numerical solution for $\varepsilon=0.1$ under the Yu (1990) model; $\cdots$, numerical solution with $\varepsilon=0$ under the SCDH model; $-\cdot \cdot-\cdot$, numerical solution with $\varepsilon=0.1$ under the SCDH model; $p=2.0$, and $\lambda=1.16$ where applicable.

eddy structure of a real plume and is sufficient to render the initial-value problem well-posed by inhibiting perturbations of arbitrarily small scales. The inclusion of this term does not significantly affect any of the published, and well-established, results for steady plumes. Similarly, the previously investigated unsteady similarity solutions for 
(a)

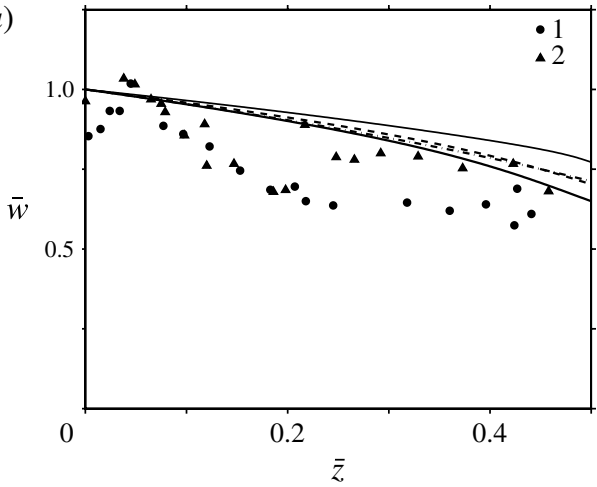

(b)

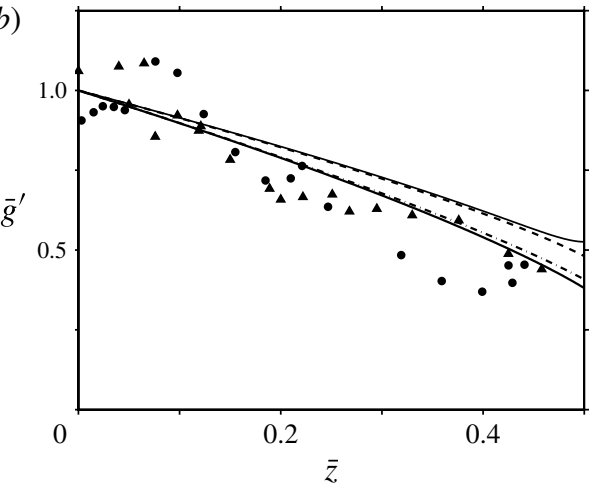

FIGURE 10. A comparison between the various models for $p=3.0, \varepsilon=0.1$ (cf. figures 7 and 8 tests 3 and 4 in Yu 1990, figure 2 in Vul'fson \& Borodin 2001): - - -, top-hat; -, Yu; - (thin), Vul'fson; - - - - Delichatsios $(\varepsilon=0)$. (a) The normalized velocity field, $\bar{w},(b)$ the normalized buoyancy field, $\bar{g}^{\prime}$. As in Vul'fson \& Borodin (2001), data points 1 and 2 refer to the data measurements taken by $\mathrm{Yu}$ (1990); the heat sources had different arrangements of packing for the combustibles.

plumes generated by a power-law (increasing) source, and those generated by a sudden bounded reduction in source strength, are largely unaffected. We suggest that this is why diffusion can be safely neglected in steady regimes and why the importance of a diffusive mechanism has not been noted previously. It is only when attempting to solve the initial-value problem for an unsteady plume subject to general conditions at its source that the ill-posed nature of the model equations becomes apparent.

Finally, our aim in this work was to revisit the range of model equations and decide which is of most relevance to fully unsteady plumes. The model of Vul'fson \& Borodin (2001) requires a plume radius that is always conical, which renders their model unsatisfactory for nonlinear changes in source conditions. The model of Delichatsios (1979) is sufficiently ill-posed that even the inclusion of diffusion is not enough to regularize it. This leaves the models of Yu (1990) and SCDH for Gaussian and top-hat plumes respectively. Both can be regularized by diffusion, and in fact, both are identical (apart from a rescaling) when the radii of velocity and reduced gravity are the same. We therefore conclude that these two models (suitably regularized) are of most relevance; there is no available experimental evidence that would distinguish between them. The regularized SCDH model is more rigorously derived and simpler, as a consequence of the top-hat assumption; however if an unsteady Gaussian plume model is required, then a regularized version of the Yu model is most appropriate.

\section{Acknowledgements}

The authors very gratefully acknowledge useful discussions with Dr C. P. Caulfield and Dr N. B. Kaye and helpful comments from Professor M. E. McIntyre and an anonymous referee. M.M.S. acknowledges funding under the University of Nottingham's Early Career Research and Knowledge Transfer Scheme.

\section{Appendix. Harmonic perturbations to unsteady jet models}

Using the notation of $\S 4$ and noting that for the definitions (4.2) we have $b=Q / \sqrt{\rho_{\infty} M}$ and $w=2 M / Q$, we seek solutions to (4.3) with $g_{0}^{\prime} \equiv 0$ that satisfy 


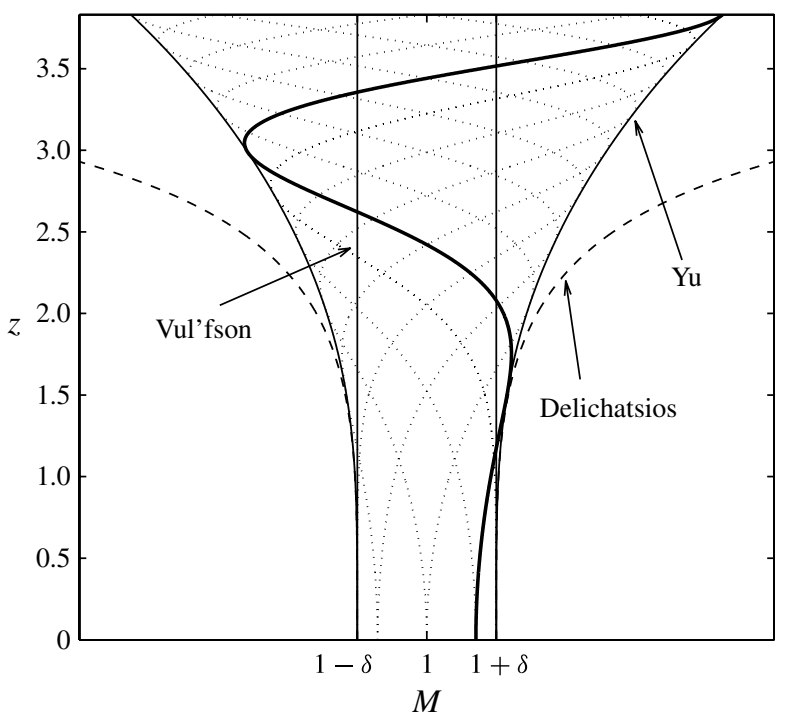

FIGURE 11. The growth of small perturbations to the momentum flux of the top-hat model and the Gaussian models of Delichatsios (1979), Yu (1990) and Vul'fson \& Borodin (2001). Qualitatively similar results as for the plume are found. The model of Delichatsios (1979) is the most unstable, while the most physically realistic in this instance is due to Vul'fson \& Borodin (2001).

the boundary condition $\boldsymbol{Q}(0, t)=M_{0}(0,1+\delta \exp \{\mathrm{i} \omega t\})^{\top}$. Writing $\boldsymbol{q}=(q, m)^{\top}$ for the perturbation vector to the steady solutions $Q_{B}=2 \alpha \rho_{\infty}^{1 / 2} M_{0}^{1 / 2} z, M_{B}=M_{0}$ it can be shown that at order $\delta$

$$
\left(\begin{array}{rr}
2 \kappa & -\kappa \\
2 & 0
\end{array}\right) \frac{\partial \boldsymbol{q}}{\partial \tau}+\frac{1}{\zeta} \frac{\partial \boldsymbol{q}}{\partial \zeta}+\frac{1}{\zeta^{2}}\left(\begin{array}{rr}
1 & -\frac{1}{2} \\
0 & 0
\end{array}\right) \boldsymbol{q}=\mathbf{0}
$$

where, as before, $\tau=\omega t$ and $\zeta=\left(z \omega / w_{B}\right)^{1 / 2}$. In the case of SCDH and Yu (1990) the models coincide up to a scaling $(\zeta \rightarrow \zeta / \sqrt{2}$ from Yu 1990 to SCDH) since there is no buoyancy. The constant $\kappa=2$, and the solution to (A 1) satisfying the prescribed boundary conditions is given by

$$
\begin{aligned}
& \left.q=\mathrm{e}^{\mathrm{i}\left[\tau-\zeta^{2}\right]}\left\{\cosh \left[\mathrm{e}^{\mathrm{i} \pi / 4} \zeta\right]-\frac{\mathrm{e}^{-\mathrm{i} \pi / 4}}{2 \zeta} \sinh \left[\mathrm{e}^{\mathrm{i} \pi / 4} \zeta\right]\right\},\right\} \\
& m=\mathrm{e}^{\mathrm{i}\left[\tau-\zeta^{2}\right]} \cosh \left[\mathrm{e}^{\mathrm{i} \pi / 4} \zeta\right] .
\end{aligned}
$$

In the far field, $\zeta \rightarrow \infty$, the solutions grow as $\exp \{\zeta / \sqrt{2}\}$.

For the Gaussian model of Delichatsios (1979), $\kappa=1$ and the solution is

$$
\begin{gathered}
q=\frac{\mathrm{e}^{\mathrm{i}\left[\tau-\zeta^{2} / 2\right]}}{2}\left(\frac{\zeta}{2}\right)^{1 / 2} \Gamma\left(\frac{3}{4}\right)\left\{\mathscr{I}_{-1 / 4}\left(\zeta^{2} / 2\right)+\mathrm{i} \mathscr{I}_{3 / 4}\left(\zeta^{2} / 2\right)\right\}, \\
m=\mathrm{e}^{\mathrm{i}\left[\tau-\zeta^{2} / 2\right]}\left(\frac{\zeta}{2}\right)^{1 / 2} \Gamma\left(\frac{3}{4}\right) \mathscr{I}_{-1 / 4}\left(\zeta^{2} / 2\right),
\end{gathered}
$$

where $\mathscr{I}$ is the modified Bessel function of the first kind. In the far field as $\zeta \rightarrow \infty$ the perturbations are more unstable than the SCDH and Yu (1990) models, as before, 
growing as $\zeta^{-1 / 2} \exp \left\{\zeta^{2} / 2\right\}$. For the Vul'fson \& Borodin (2001) model the solutions are given by

$$
q=\frac{1}{2} \mathrm{e}^{\mathrm{i}\left[\tau-\zeta^{2} / 2\right]}, \quad m=\mathrm{e}^{\mathrm{i}\left[\tau-\zeta^{2} / 2\right]},
$$

and the solutions neither grow nor decay.

Figure 11 illustrates the growth of the asymptotically small perturbations to the source conditions of the jet under the four models considered. Qualitatively similar responses to those found in the plume configuration are found. The Vul'fson \& Borodin (2001) model neither grows nor decays and so exhibits the most physically realistic response in terms of perturbation growth. In the absence of any diffusion the scalably identical models of SCDH and Yu (1990) (due to the vanishing reduced gravity radius) show the same ill-posed behaviour, with perturbations growing exponentially with downstream distance from the source. Again the perturbations grow most rapidly under the model of Delichatsios (1979). As with the plume modelling, no finite dominant frequency arises in the Delichatsios (1979), Yu (1990) or SCDH models and so they are ill-posed.

\section{REFERENCES}

Abramowitz, M. \& Stegun (Eds) 1965 Handbook of Mathematical Functions with Formulas, Graphs and Mathematical Tables. Dover.

Delichatsios, 1979 Time similarity analysis of unsteady buoyant plumes in neutral surroundings. J. Fluid Mech. 93, 241-250.

Hewitt, R. E. \& Duck, P. W. 2011 Pulsatile jets. J. Fluid Mech. 670, 240-259.

Holland, P. R. 2011 Oscillating dense plumes. J. Phys. Oceanogr. 41 (8), 1465-1483.

HUNT, G. R. \& VAN DEN BREMER, T. S. 2010 Classical plume theory: 1937-2010 and beyond. IMA J. Appl. Maths 76 (3), 424-448.

Jospen, D. \& SAUt, J. C. 1990 Short-wave instabilities and ill-posed initial-value problems. Theor. Comput. Fluid Dyn. 1, 191-227.

Kaminski, E., TAit, S. \& CARAZZO, G. 2005 Turbulent entrainment in jets with arbitrary buoyancy. J. Fluid Mech. 526, 361-376.

KAYE, N. B. 2008 Turbulent plumes in stratified environments: A review of recent work. Atmos.-Ocean 46 (4), 433-441.

Morton, B. R., TAYlor, G. I. \& TURner, J. S. 1956 Buoyant gravitational convection from instantaneous and maintained point sources. Proc. R. Soc. Lond. A 234, 1-23.

PAPANicolaOU, P. N. \& List, E. J. 1988 Investigations of round vertical buoyant jets. J. Fluid Mech. 195, 341-391.

PrandtL, L. 1952 The Essentials of Fluid Dynamics. Blackie \& Son Ltd.

Ricou, F. P. \& SPAlding, D. B. 1961 Measurements of entrainment by axisymmetric turbulent jets. J. Fluid Mech. 8, 21-32.

Rooney, G. G. \& Linden, P. F. 1996 Similarity considerations for non-Boussinesq plumes in an unstratified environment. J. Fluid Mech. 318, 237-250.

Rouse, H., Yih, C. S. \& HumphreYs, H. W. 1952 Gravitational convection from a boundary source. Tellus 4, 201-210.

SCASE, M. M. 2009 Evolution of volcanic eruption columns. J. Geophys. Res. 114, F04003.

Scase, M. M., Aspden, A. J. \& CAulfield, C. P. 2009 The effect of sudden source buoyancy flux increases on turbulent plumes. J. Fluid Mech. 635, 137-169.

Scase, M. M., Caulfield, C. P. \& Dalziel, S. B. $2006 a$ Boussinesq plumes with decreasing source strengths in stratified environments. J. Fluid Mech. 563, 463-472.

Scase, M. M., Caulfield, C. P. \& Dalziel, S. B. 2008 Temporal variation of non-ideal plumes with sudden reductions in buoyancy flux. J. Fluid Mech. 600, 181-199.

Scase, M. M., Caulfield, C. P., Dalziel, S. B. \& Hunt, J. C. R. $2006 b$ Time-dependent plumes and jets with decreasing source strengths. J. Fluid Mech. 563, 443-461. 
Scase, M. M., Caulfield, C. P., Linden, P. F. \& Dalziel, S. B. 2007 Local implications for self-similar turbulent plume models. J. Fluid Mech. 575, 257-265.

SCHMidT, F. H. 1957 On the diffusion of heated jets. Tellus 9, 378-383.

Schmidt, W. 1941 Turbulente Ausbreitung eines Stromes erhitzter Luft. Z. Angew. Math. Mech. 21 (6), 351-363.

TURner, J. S. 1962 The 'starting plume' in neutral surroundings. J. Fluid Mech. 13, 356-368.

VUL'FSON, A. N. 2001a Convective-region top front propagation in a uniform medium under the action of point, linear, and plane heat and momentum sources. Fluid Dyn. 36 (3), 418-428 (translation from Izv. Ross. Acad. Nauk, Mekh. Zhid. i Gaza (3), 90-101).

VUL'FSON, A. N. $2001 b$ Unsteady self-similar convection from a point source of heat and passive tracer in a neutral atmosphere. Russ. Met. Hyd. 1, 23-35.

Vul'Fson, A. N. \& Borodin, O. O. 2001 Self- similar propagation regimes of a non stationary high temperature convective jet in the adiabatic atmosphere. J. Appl. Mech. Tech. Phys. 42 (2), 255-261.

YU, H.-Z. 1990 Transient plume influence in measurement of convective heat release rates of fast-growing fires using a large-scale fire products collector. Trans. ASME 112, 186-191. 